\title{
Steady flows of a highly rarefied gas induced by nonuniform wall temperature
}

\section{AUTHOR(S):}

Kosuge, Shingo; Aoki, Kazuo; Takata, Shigeru; Hattori, Ryosuke; Sakai, Daisuke

\section{CITATION:}

Kosuge, Shingo ...[et al]. Steady flows of a highly rarefied gas induced by nonuniform wall temperature. Physics of Fluids 2011, 23(3): 030603.

ISSUE DATE:

2011-03

URL:

http://hdl.handle.net/2433/159436

RIGHT:

(C) 2011 American Institute of Physics 


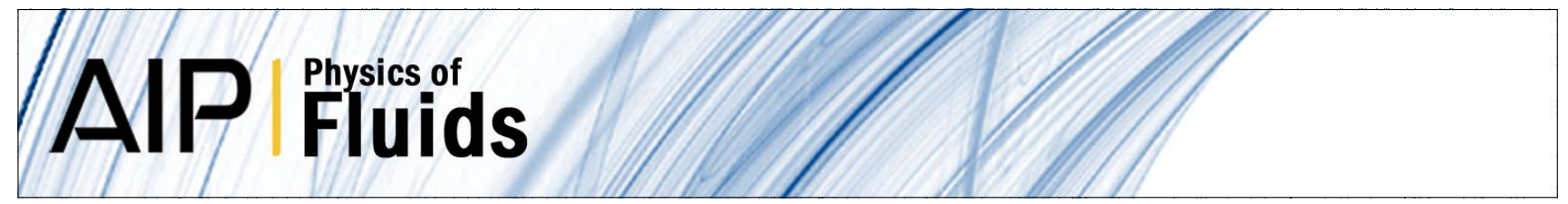

\section{Steady flows of a highly rarefied gas induced by nonuniform wall temperature}

Shingo Kosuge, Kazuo Aoki, Shigeru Takata, Ryosuke Hattori, and Daisuke Sakai

Citation: Phys. Fluids 23, 030603 (2011); doi: 10.1063/1.3558872

View online: http://dx.doi.org/10.1063/1.3558872

View Table of Contents: http://pof.aip.org/resource/1/PHFLE6/v23/i3

Published by the American Institute of Physics.

\section{Related Articles}

Dissipation from microscale and nanoscale beam resonators into a surrounding fluid Appl. Phys. Lett. 92, 124102 (2008)

Modeling of associative ionization reactions in hypersonic rarefied flows

Phys. Fluids 19, 096102 (2007)

Gas flow near a plate oscillating longitudinally with an arbitrary frequency

Phys. Fluids 19, 017110 (2007)

Formulation and numerical analysis of diatomic molecular dissociation using Boltzmann kinetic equation Phys. Fluids 19, 017103 (2007)

A direct simulation Monte Carlo method for rarefied gas flows in the limit of small Mach number

Phys. Fluids 17, 107107 (2005)

\section{Additional information on Phys. Fluids}

Journal Homepage: http://pof.aip.org/

Journal Information: http://pof.aip.org/about/about_the_journal

Top downloads: http://pof.aip.org/features/most_downloaded

Information for Authors: http://pof.aip.org/authors

\section{ADVERTISEMENT}

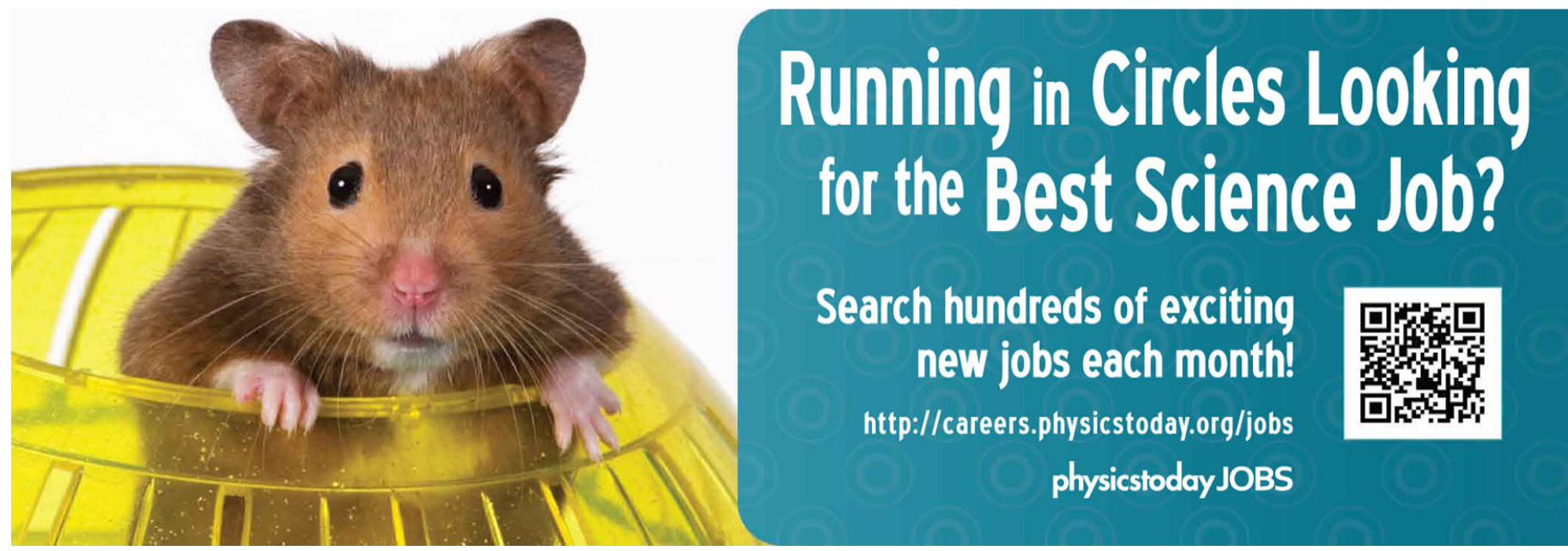




\title{
Steady flows of a highly rarefied gas induced by nonuniform wall temperature
}

\author{
Shingo Kosuge, ${ }^{\text {a) }}$ Kazuo Aoki, Shigeru Takata, Ryosuke Hattori, and Daisuke Sakai \\ Department of Mechanical Engineering and Science and Advanced Research Institute of Fluid Science and \\ Engineering, Graduate School of Engineering, Kyoto University, Kyoto 606-8501, Japan
}

(Received 3 October 2010; accepted 17 January 2011; published online 18 March 2011)

\begin{abstract}
Steady behavior of a rarefied gas between parallel plates with sinusoidal temperature distribution is investigated on the basis of the Boltzmann equation. The Cercignani-Lampis (CL) model or the Lord model for diffuse scattering with incomplete energy accommodation is adopted as the boundary condition on the plates. Most of the analysis is carried out numerically with special interest in the free-molecular limit. In the case of the CL model, the nonuniform temperature distribution of the plates may induce a steady free-molecular flow, which is in contrast with the earlier results for the Maxwell-type model [Y. Sone, J. Méc. Théor. Appl. 3, 315 (1984); J. Méc. Théor. Appl. 4, 1 (1985)]. This fact is confirmed through an accurate deterministic computation based on an integral equation. In addition, computations for a wide range of parameters by means of the direct simulation Monte Carlo method reveal that the flow field changes according to the accommodation coefficients and is classified into four types. The effect of intermolecular collisions on the flow is also examined. In the case of the Lord model, no steady flow of the free-molecular gas is induced as in the case of the Maxwell-type model. This result is extended to the case of a more general boundary condition that gives the cosine law (Lambert's law) for the reflected molecular flux. (C) 2011 American Institute of Physics. [doi:10.1063/1.3558872]
\end{abstract}

\section{INTRODUCTION}

Let us consider a gas around a solid body (or bodies) at rest, whose surface temperature is time-independent but spatially nonuniform. In the framework of continuum gas dynamics (or the Navier-Stokes set of equations), no steady flow is expected, if an external force, such as gravity, is absent. In contrast, in the case of rarefied gases, steady flows may be induced around the body even in the absence of an external force. This type of flow, induced solely by the effect of steady temperature field, is peculiar to rarefied gases and has been an intriguing research subject in rarefied (or molecular) gas dynamics (see, e.g., Refs. 1-4). The most famous example would be the thermal-creep flow $^{5-8}$ of a slightly rarefied gas, which is induced along a surface with nonuniform temperature in the direction of the temperature gradient. Some other examples, such as the thermal-stress slip flow, ${ }^{9,10}$ nonlinear-thermal-stress flow, ${ }^{11}$ thermal-edge flow, ${ }^{12,13}$ and so on, have also been recognized and closely studied on the basis of the Boltzmann equation.

Meanwhile, Sone revealed in Refs. 14 and 15 (see also Refs. 2, 4, and 16) an interesting and nontrivial feature of the abovementioned type of steady flows in the limit of a freemolecular gas (i.e., the gas so highly rarefied that intermolecular collisions can be neglected). When the gas-surface interaction on the bodies is described by the so-called Maxwell-type boundary condition (i.e., a linear combination of the diffuse-reflection and the specular-reflection condition), the steady flow due to the thermal effect vanishes in the free-molecular limit, irrespective of the temperature dis-

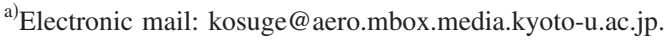

tribution of the bodies (if an unbounded domain is considered, the state of the gas at infinity must satisfy a certain condition detailed in Refs. 4, 14, and 15). This is not an obvious fact at all. This discovery is based on an analytical solution that was obtained by Sone and describes the effect of the boundary temperature in a very general setting. The analytical solution was then applied to obtain, analytically and numerically, the forces acting on heated (or cooled) bodies in a free-molecular gas in various geometries (see Ref. 4). The results in Refs. 14 and 15 were revisited some time later from the viewpoint of functional analysis in Ref. 17. It should also be mentioned that the vanishing of a flow in a similar situation was investigated in Ref. 18.

The present study is motivated by a natural question whether or not a steady flow is induced by a steady temperature field in the free-molecular limit if a boundary condition other than the Maxwell-type condition is employed. The Maxwell-type condition is one of the fundamental models for gas-surface interaction and has been widely used in the literature. At the same time, some drawbacks have been pointed out and thus, efforts to devise a better model have been made, e.g., in Refs. 19-22. In particular, the Cercignani-Lampis (CL) model $^{22}$ successfully overcomes one major drawback of the Maxwell-type model: by adjusting the two accommodation coefficients involved in it, the CL model can reproduce well the actual data for the distribution of scattered molecules obtained in molecular beam scattering experiments (see Refs. 23 and 24). This is mostly impossible for the Maxwell-type model. The CL model has often been used as a realistic boundary condition in the literature, especially in recent years (see, e.g., Refs. 25-29).

In the present study, we consider a rarefied gas between 
parallel plates with sinusoidal temperature distribution and investigate the steady behavior of the gas on the basis of the Boltzmann equation and the CL boundary condition. As mentioned above, we are particularly interested in the freemolecular limit. Therefore, we first carry out an accurate deterministic computation using an integral equation for the free-molecular limit derived from the Boltzmann equation and the boundary condition. Unlike the case of the Maxwelltype condition, the results for some typical cases demonstrate nonvanishing gas flows in the free-molecular limit. Then we carry out computations by means of the direct simulation Monte Carlo (DSMC) method ${ }^{30,31}$ for a wide range of parameters, i.e., the Knudsen number $[=($ molecular mean free path $) /($ distance between the plates $)]$ as well as the accommodation coefficients involved in the CL model. The DSMC computation for the free-molecular limit complements the results obtained by the integral equation.

For comparison, we also investigate the case of the Lord model for diffuse scattering with incomplete energy accommodation $^{32}$ and show that the flow vanishes in the free-molecular limit, as in the case of the Maxwell-type condition. As an extension of the Lord model, we consider a more general boundary condition that gives the cosine law (Lambert's law) for the reflected molecular flux (i.e., the case where the reflected molecular flux is proportional to the cosine of the angle between flux's direction and the surface normal) in the general configuration, as in Refs. 14 and 15, and show that for such a boundary condition, the flow due to a steady temperature field vanishes in the free-molecular limit.

In the present paper, the abovementioned type of boundary condition, which gives the cosine law for the reflected molecular flux, will be referred to as the Lambertian condition. Equivalently, the Lambertian condition may also be defined as a condition which always yields the isotropic velocity distribution of reflected molecules regardless of the incoming distribution (the above "isotropic" means that the distribution is independent of the direction of the molecular velocity). The diffuse-reflection condition is, of course, a member of the Lambertian conditions. To avoid confusion, it should be noted here that the "isotropic surface" (or "isotropic boundary condition") appearing in the literature is a different and more general concept, which means that the scattering properties of the surface are invariant under the rotation around the normal.

This paper is dedicated to the memory of Carlo Cercignani who passed away recently. He had been a leader in the field of kinetic theory for almost a half century and had made outstanding contributions to the field. It is remarkable that his about 300 papers cover all aspects of kinetic theory: mathematical, physical, engineering, and numerical aspects. Several books he authored have been a guideline and a fountain of knowledge for researchers and students for a long time. He published, with Maria Lampis, many important papers on physics of rarefied gases. One of them is the celebrated CL model for gas-surface interaction, to which the present paper is related.

\section{FORMULATION}

\section{A. Problem and assumptions}

Consider a rarefied gas between two parallel plates located at $X_{2}= \pm L / 2$, where $X_{i}(i=1,2,3)$ is the space rectangular coordinate. Both plates are kept at the same temperature $T_{w}$, where $T_{w}$ is a sinusoidal function of $X_{1}$ written as $T_{w}=T_{0}\left[1+\tau \sin \left(2 \pi X_{1} / \lambda L\right)\right]$ with $T_{0}$ being the reference temperature and $\tau(|\tau|<1)$ and $\lambda$ being dimensionless constants. We investigate steady behavior of the gas under the following assumptions: (i) the behavior of the gas is described by the Boltzmann equation; (ii) intermolecular collisions are elastic and are described by the hard-sphere model; and (iii) gas-surface interaction on the plates is described by the CL model or by the Lord model for diffuse scattering with incomplete energy accommodation. For the sake of simplicity, the accommodation coefficients involved in the boundary condition [see Eqs. (6) and (8) below] are assumed to be independent of $T_{w}$ and uniform throughout the boundaries.

Let us summarize the main notation used in the paper. The symbols $m$ and $d$ denote the molecular mass and diameter, respectively; $\rho_{0}$ is the average density of the gas, and $p_{0}$ is the reference pressure defined as $p_{0}=(k / m) \rho_{0} T_{0}$, where $k$ is the Boltzmann constant; $x_{i}$ denotes the dimensionless space coordinate defined as $x_{i}=X_{i} / L$, and $\left(2 k T_{0} / m\right)^{1 / 2} \zeta_{i}$ [or $\left.\left(2 k T_{0} / m\right)^{1 / 2} \zeta\right]$ denotes the molecular velocity; and $\rho_{0}\left(2 k T_{0} / m\right)^{-3 / 2} f\left(x_{1}, x_{2}, \zeta\right)$ is the velocity distribution function of the molecules. The macroscopic quantities such as the density $\rho_{0} \hat{\rho}$, flow velocity $\left(2 k T_{0} / m\right)^{1 / 2} u_{i}$ [or $\left(2 k T_{0} / m\right)^{1 / 2} \boldsymbol{u}$, pressure $p_{0} \hat{p}$, and temperature $T_{0} \hat{T}$ are defined in terms of the moment of $f$ as follows:

$$
\begin{aligned}
& \hat{\rho}=\int f \mathrm{~d} \boldsymbol{\zeta}, \quad \hat{\rho} \boldsymbol{u}=\int \boldsymbol{\zeta} f \mathrm{~d} \boldsymbol{\zeta}, \\
& \hat{p}=\hat{\rho} \hat{T}=\frac{2}{3} \int|\boldsymbol{\zeta}-\boldsymbol{u}|^{2} f \mathrm{~d} \zeta,
\end{aligned}
$$

where $\mathrm{d} \zeta=\mathrm{d} \zeta_{1} \mathrm{~d} \zeta_{2} \mathrm{~d} \zeta_{3}$, and the domain of integration is the whole space of $\zeta$.

\section{B. Basic equation and boundary condition}

By the use of the notation introduced above, the Boltzmann equation for the present spatially two-dimensional problem may be written in the following dimensionless form (see, e.g., Ref. 4):

$$
\zeta_{1} \frac{\partial f}{\partial x_{1}}+\zeta_{2} \frac{\partial f}{\partial x_{2}}=\frac{1}{\mathrm{Kn}} Q[f] .
$$

Here, $Q$ is the collision term for hard-sphere molecular gases and is written as

$$
\begin{aligned}
& Q[f]=\frac{1}{2 \sqrt{2} \pi} \int\left(f^{\prime} f_{*}^{\prime}-f f_{*}\right)|\boldsymbol{e} \cdot \boldsymbol{V}| \mathrm{d} \Omega(\boldsymbol{e}) \mathrm{d} \boldsymbol{\zeta}_{*}, \\
& f=f\left(x_{1}, x_{2}, \zeta\right), \quad f_{*}=f\left(x_{1}, x_{2}, \boldsymbol{\zeta}_{*}\right), \\
& f^{\prime}=f\left(x_{1}, x_{2}, \boldsymbol{\zeta}^{\prime}\right), \quad f_{*}^{\prime}=f\left(x_{1}, x_{2}, \boldsymbol{\zeta}_{*}^{\prime}\right),
\end{aligned}
$$




$$
\begin{aligned}
& \boldsymbol{\zeta}^{\prime}=\boldsymbol{\zeta}+(\boldsymbol{e} \cdot \boldsymbol{V}) \boldsymbol{e}, \quad \boldsymbol{\zeta}_{*}^{\prime}=\boldsymbol{\zeta}_{*}-(\boldsymbol{e} \cdot \boldsymbol{V}) \boldsymbol{e}, \\
& \boldsymbol{V}=\boldsymbol{\zeta}_{*}-\boldsymbol{\zeta},
\end{aligned}
$$

where $\mathrm{d} \zeta_{*}=\mathrm{d} \zeta_{* 1} \mathrm{~d} \zeta_{{ }_{*} 2} \mathrm{~d} \zeta_{* 3}, \boldsymbol{e}$ is a unit vector, and $\mathrm{d} \Omega(\boldsymbol{e})$ is the solid-angle element in the direction of $\boldsymbol{e}$. The integration in Eq. (3a) is carried out over the whole direction of $\boldsymbol{e}$ and over the whole space of $\boldsymbol{\zeta}_{*}$. The $\mathrm{Kn}$ in Eq. (2) is the Knudsen number defined as

$$
\mathrm{Kn}=l_{0} / L, \quad l_{0}=\left[\sqrt{2} \pi d^{2}\left(\rho_{0} / m\right)\right]^{-1},
$$

where $l_{0}$ is the reference mean free path of the gas molecules at an equilibrium state at rest at density $\rho_{0}$.

The boundary condition on the plates may be written in terms of the scattering kernel $R$ as

$$
\begin{aligned}
f\left(x_{1}, \pm \frac{1}{2}, \boldsymbol{\zeta}\right)= & \int_{\mp \zeta_{* 2}<0}\left|\frac{\boldsymbol{\zeta}_{* 2}}{\zeta_{2}}\right| R\left(\boldsymbol{\zeta}_{*}, \boldsymbol{\zeta}, x_{1}\right) \\
& \times f\left(x_{1}, \pm \frac{1}{2}, \boldsymbol{\zeta}_{*}\right) \mathrm{d} \boldsymbol{\zeta}_{*}, \text { for } \mp \zeta_{2}>0,
\end{aligned}
$$

where the upper and lower signs correspond to $x_{2}=1 / 2$ and $x_{2}=-1 / 2$, respectively.

In the case of the CL model, ${ }^{22}$ the scattering kernel $R$ appearing in the boundary condition (5) is given by

$$
\begin{aligned}
& R=R_{n}\left(\zeta_{* 2}, \zeta_{2}\right) R_{t}\left(\zeta_{* 1}, \zeta_{1}\right) R_{t}\left(\zeta_{* 3}, \zeta_{3}\right), \\
& R_{n}\left(c_{*}, c\right)=\frac{2}{\alpha_{n} \hat{T}_{w}}|c| I_{0}\left(\frac{2\left(1-\alpha_{n}\right)^{1 / 2}}{\alpha_{n} \hat{T}_{w}} c_{*} c\right) \\
& \times \exp \left[-\frac{c^{2}+\left(1-\alpha_{n}\right) c_{*}^{2}}{\alpha_{n} \hat{T}_{w}}\right], \\
& R_{t}\left(c_{*}, c\right)=\frac{1}{\left[\pi \alpha_{t}\left(2-\alpha_{t}\right) \hat{T}_{w}\right]^{1 / 2}} \\
& \times \exp \left\{-\frac{\left[c-\left(1-\alpha_{t}\right) c_{*}\right]^{2}}{\alpha_{t}\left(2-\alpha_{t}\right) \hat{T}_{w}}\right\}, \\
& I_{0}(y)=\frac{1}{\pi} \int_{0}^{\pi} \exp (y \cos \theta) \mathrm{d} \theta,
\end{aligned}
$$

where the argument $x_{1}$ is omitted for simplicity. In Eq. (6), $I_{0}$ is the modified Bessel function ${ }^{33}$ of first kind and zeroth order, $\hat{T}_{w}\left(\equiv T_{w} / T_{0}\right)$ is the dimensionless surface temperature, i.e.,

$$
\hat{T}_{w}=1+\tau \sin \left(2 \pi x_{1} / \lambda\right),
$$

$\alpha_{n}\left(0 \leq \alpha_{n} \leq 1\right)$ is the accommodation coefficient for partial kinetic energy associated with a molecular velocity component normal to the boundary, and $\alpha_{t}\left(0 \leq \alpha_{t} \leq 2\right)$ is that for tangential momentum. Incidentally, the accommodation coefficient for partial energy associated with the tangential velocity component is given by $\alpha_{t}\left(2-\alpha_{t}\right)$; if $\alpha_{n}=\alpha_{t}\left(2-\alpha_{t}\right)$ $(\equiv \sigma), \sigma$ corresponds to the accommodation coefficient for total kinetic energy. The CL model reduces to the diffuse- reflection condition when $\left(\alpha_{n}, \alpha_{t}\right)=(1,1)$ and to the specularreflection condition when $\left(\alpha_{n}, \alpha_{t}\right)=(0,0)$. When $\alpha_{t}>1$, more than half of impinging molecules experience the back scattering (the scattering where the change in the direction of tangential velocity between before and after the reflection is larger than $\pi / 2){ }^{34}$ The extreme case $\left(\alpha_{n}, \alpha_{t}\right)=(0,2)$ corresponds to the reverse reflection, where an impinging molecule with velocity $\boldsymbol{\zeta}_{*}$ is reemitted with velocity $-\boldsymbol{\zeta}_{*}$.

The Lord model for diffuse scattering with incomplete accommodation was devised in Ref. 32 as one of important variations of the $\mathrm{CL}$ model. The scattering kernel for this model is given by

$$
\begin{aligned}
& R=\frac{2\left[\sigma(1-\sigma)^{1 / 2}\right]^{-1}}{\pi \hat{T}_{w}} \frac{\left|\zeta_{2}\right|}{\zeta_{*} \zeta} I_{1}\left(\frac{2(1-\sigma)^{1 / 2}}{\sigma \hat{T}_{w}} \zeta_{*} \zeta\right) \\
& \times \exp \left[-\frac{\zeta^{2}+(1-\sigma) \zeta_{*}^{2}}{\sigma \hat{T}_{w}}\right], \\
& \zeta=|\zeta|, \quad \zeta_{*}=\left|\zeta_{*}\right|, \\
& I_{1}(y)=\frac{1}{\pi} \int_{0}^{\pi} \exp (y \cos \theta) \cos \theta \mathrm{d} \theta,
\end{aligned}
$$

where $I_{1}$ is the modified Bessel function ${ }^{33}$ of first kind and first order and $\sigma(0 \leq \sigma \leq 1)$ is the (total) energy accommodation coefficient. The case $\sigma=0$ corresponds to the elastic diffuse scattering and the case $\sigma=1$ corresponds to the ordinary diffuse reflection (with perfect accommodation). In any case, the velocity distribution of reflected molecules derived from Eqs. (5) and (8a) is isotropic (i.e., independent of the direction of $\zeta$ ) and thus the reflected molecular flux always satisfies the cosine law. Therefore the Lord model is a member of the Lambertian conditions (see the next-to-last paragraph in Sec. I).

Let us consider the special case where the equality $\sigma$ $=\alpha_{n}=\alpha_{t}\left(2-\alpha_{t}\right)$ holds. Then, the CL model (6) and the Lord model (8) give the same energy accommodation coefficient. In addition, if the velocity distribution of impinging molecules is the stationary Maxwellian (with arbitrary temperature), the two models yield the same distribution for reflected molecules. In this sense, the two models satisfying the above equality are relatively close to each other. However, the CL model is not Lambertian in general even when the equality holds, unless $\alpha_{n}=\alpha_{t}=1$.

It is readily seen that the present boundary-value problem, i.e., Eqs. (2) and (5), is characterized by the following five or four dimensionless parameters:

$$
\left\{\begin{array}{lll}
\mathrm{Kn}, & \tau, \lambda, \quad \alpha_{n}, \quad \alpha_{t}, & \text { for the CL model, } \\
\mathrm{Kn}, \quad \tau, \quad \lambda, \quad \sigma, & \text { for the Lord model. }
\end{array}\right.
$$

As explained in Sec. I, our main interest is in the case of the free-molecular gas $(\mathrm{Kn} \rightarrow \infty)$, which will be considered in more detail in Sec. II C.

Finally, we should note that the following relations hold because of the mirror symmetry (with respect to $x_{2}=0$ and $\left.x_{1}= \pm \lambda / 4\right)$ of the problem:

$$
f\left(x_{1}, x_{2}, \zeta_{1}, \zeta_{2}, \zeta_{3}\right)=f\left(x_{1},-x_{2}, \zeta_{1},-\zeta_{2}, \zeta_{3}\right),
$$




$$
\begin{aligned}
& f\left(x_{1}, x_{2}, \zeta_{1}, \zeta_{2}, \zeta_{3}\right)=f\left(\frac{\lambda}{2}-x_{1}, x_{2},-\zeta_{1}, \zeta_{2}, \zeta_{3}\right), \\
& f\left(x_{1}, x_{2}, \zeta_{1}, \zeta_{2}, \zeta_{3}\right)=f\left(-\frac{\lambda}{2}-x_{1}, x_{2},-\zeta_{1}, \zeta_{2}, \zeta_{3}\right) .
\end{aligned}
$$

Therefore, we can solve the problem in a closed rectangular domain $\left|x_{1}\right| \leq \lambda / 4$ and $0 \leq x_{2} \leq 1 / 2$, imposing Eq. (5) with upper signs on $x_{2}=1 / 2$ and the specular-reflection condition at $x_{2}=0$ [i.e., Eq. (9a) with $x_{2}=0$ for $\zeta_{2}>0$ ] and at $x_{1}$ $= \pm \lambda / 4$ [i.e., Eq. (9b) with $x_{1}=\lambda / 4$ for $\zeta_{1}<0$ and Eq. (9c) with $x_{1}=-\lambda / 4$ for $\left.\zeta_{1}>0\right]$.

\section{Integral equation for the free-molecular gas}

Let us consider the case when the gas is free-molecular, i.e., the limit where $\mathrm{Kn} \rightarrow \infty$. In this limit, the right-hand side (RHS) of the Boltzmann equation (2) vanishes and thus, the velocity distribution function $f$ for fixed $\zeta$ does not change along a flight path of a molecule with velocity $\zeta$ [or along the characteristics of Eq. (2)]. Tracing back the flight path to the boundaries and taking account of Eq. (9), we can derive the following relation for $f$. For $\left|x_{1}\right| \leq \lambda / 4$ and $0 \leq x_{2} \leq 1 / 2$,

$$
\begin{aligned}
& f\left(x_{1}, x_{2}, \zeta_{1}, \zeta_{2}, \zeta_{3}\right)
\end{aligned}
$$

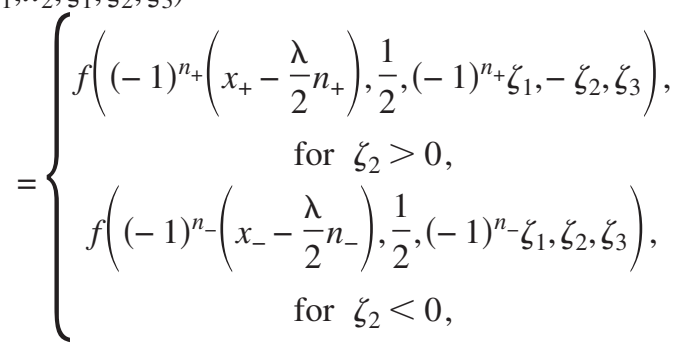

with

$$
\begin{aligned}
& x_{ \pm}\left(x_{1}, x_{2}, \zeta_{1}, \zeta_{2}\right)=x_{1} \mp\left(\frac{1}{2} \pm x_{2}\right) \frac{\zeta_{1}}{\zeta_{2}}, \\
& n_{ \pm}\left(x_{1}, x_{2}, \zeta_{1}, \zeta_{2}\right)=\left\lfloor\frac{1}{2}+\frac{2}{\lambda} x_{ \pm}\right\rfloor .
\end{aligned}
$$

Here, the upper (lower) signs go together and $\lfloor x\rfloor$ is the largest integer not greater than $x$ (the floor function). Since the inequality $\left|x_{ \pm}-(\lambda / 2) n_{ \pm}\right| \leq \lambda / 4$ holds, Eq. (10) means that the velocity distribution $f$ at arbitrary point $\left(x_{1}, x_{2}\right)$ for arbitrary $\zeta$ (but $\zeta_{2} \neq 0$ ) is expressed in terms of $f$ for reflected molecules $\left(\zeta_{2}<0\right)$ at the upper plate $\left(x_{2}=1 / 2\right)$ in the interval $\left|x_{1}\right| \leq \lambda / 4$.

The boundary condition (5) at $x_{2}=1 / 2$ and Eq. (10) yield

$$
\begin{aligned}
f\left(x_{1}, \frac{1}{2}, \zeta\right)= & \int_{\zeta_{* 2}<0}\left|\frac{\zeta_{* 2}}{\zeta_{2}}\right| R\left(\zeta_{*}, \zeta, x_{1}\right) \\
& \times f\left((-1)^{n}\left(x-\frac{\lambda}{2} n\right), \frac{1}{2},(-1)^{n} \zeta_{* 1}, \zeta_{* 2}, \zeta_{* 3}\right) \mathrm{d} \boldsymbol{\zeta}_{*},
\end{aligned}
$$

for $\zeta_{2}<0$, $x=x_{1}+\frac{\zeta_{* 1}}{\zeta_{* 2}}, \quad n=\left\lfloor\frac{1}{2}+\frac{2}{\lambda} x\right\rfloor$.

Equation (12) is a linear homogeneous integral equation for $f$ for $\left|x_{1}\right| \leq \lambda / 4, x_{2}=1 / 2$, and $\zeta_{2}<0$. Once its solution is obtained, the distribution $f$ in the whole gas region can be derived by the use of Eq. (10). Here we should note that Eq. (12) determines the solution up to a multiplicative constant. The unique solution is obtained by specifying the amount of the gas contained in the domain (in the present problem, the average density $\rho_{0}$ is specified in Sec. II A).

In the case of the CL scattering kernel (6), one can eliminate the independent variable $\zeta_{3}$ by considering the following marginal distribution functions $g$ and $h$ :

$$
g=\int_{-\infty}^{\infty} f \mathrm{~d} \zeta_{3}, \quad h=\int_{-\infty}^{\infty} \zeta_{3}^{2} f \mathrm{~d} \zeta_{3} .
$$

The macroscopic quantities in Eq. (1) are rewritten in terms of $g$ and $h$ as

$$
\begin{aligned}
& \hat{\rho}=\int g \mathrm{~d} \zeta_{1} \mathrm{~d} \zeta_{2}, \quad \hat{\rho}\left[\begin{array}{l}
u_{1} \\
u_{2}
\end{array}\right]=\int\left[\begin{array}{l}
\zeta_{1} \\
\zeta_{2}
\end{array}\right] g \mathrm{~d} \zeta_{1} \mathrm{~d} \zeta_{2}, \\
& \hat{p}=\hat{\rho} \hat{T}=\frac{2}{3} \int\left[\left(\zeta_{1}^{2}+\zeta_{2}^{2}\right) g+h\right] \mathrm{d} \zeta_{1} \mathrm{~d} \zeta_{2}-\frac{2}{3} \hat{\rho}\left(u_{1}^{2}+u_{2}^{2}\right),
\end{aligned}
$$

where $u_{3} \equiv 0$ is assumed. The integral equation for $g$ or $h$ is obtained by integrating both sides of Eq. (12a) multiplied by 1 or $\zeta_{3}^{2}$ with respect to $\zeta_{3}$. As seen in Eq. (6), the scattering kernel for the CL model is decomposed into a product of three factors for each velocity component and the integration of the factor containing $\zeta_{3}$ is easy. We readily obtain the following result. For $\zeta_{2}<0$,

$$
\begin{aligned}
g\left(x_{1}, \frac{1}{2}, \zeta_{1}, \zeta_{2}\right)= & \mathcal{J}[g]\left(x_{1}, \zeta_{1}, \zeta_{2}\right), \\
h\left(x_{1}, \frac{1}{2}, \zeta_{1}, \zeta_{2}\right)= & \left(1-\alpha_{t}\right)^{2} \mathcal{J}[h]\left(x_{1}, \zeta_{1}, \zeta_{2}\right) \\
& +\frac{1}{2} \alpha_{t}\left(2-\alpha_{t}\right) \hat{T}_{w}\left(x_{1}\right) g\left(x_{1}, \frac{1}{2}, \zeta_{1}, \zeta_{2}\right) .
\end{aligned}
$$

The operator $\mathcal{H} \cdot]$ is defined as

$$
\begin{aligned}
\mathcal{J}[g]= & \int_{\zeta_{* 2}<0}\left|\frac{\zeta_{* 2}}{\zeta_{2}}\right| R_{n}\left(\zeta_{* 2}, \zeta_{2}, x_{1}\right) R_{t}\left(\zeta_{* 1}, \zeta_{1}, x_{1}\right) \\
& \times g\left((-1)^{n}\left(x-\frac{\lambda}{2} n\right), \frac{1}{2},(-1)^{n} \zeta_{* 1}, \zeta_{* 2}\right) \mathrm{d} \zeta_{* 1} \mathrm{~d} \zeta_{* 2},
\end{aligned}
$$

with $x$ and $n$ being defined in Eq. (12b). Once the solution of Eq. (15) (i.e., $g$ and $h$ for $\left|x_{1}\right| \leq \lambda / 4, x_{2}=1 / 2$, and $\zeta_{2}<0$ ) is obtained, we can derive $g$ and $h$ in the whole gas region by using essentially the same relation as Eq. (10) and can obtain the macroscopic quantities by using Eq. (14). 
In the case of the Lord scattering kernel (8), the above elimination of $\zeta_{3}$ is impossible. Instead, it is possible to prove the absence of any steady free-molecular flow as will be discussed later in Sec. III B.

\section{NUMERICAL ANALYSIS AND RESULTS}

In this section, we carry out the direct numerical analysis of the problem formulated in Sec. II and show its results. The temperature distribution of the plates is fixed as

$$
\tau=0.5, \quad \lambda=2,
$$

throughout the whole analysis.

In the case of the CL model (6), two different methods are used in the numerical analysis: a deterministic computation for the integral equation (15) for the free-molecular gas $(\mathrm{Kn} \rightarrow \infty)$ and a stochastic computation using the DSMC method $^{30,31}$ for the boundary-value problem [i.e., Eqs. (2) and (5)] for wide range of $\mathrm{Kn}$ (including $\mathrm{Kn} \rightarrow \infty$ ). The former is more precise but needs much more CPU time. Thus, the former is performed only for several values of $\alpha_{n}$ and $\alpha_{t}$ to confirm the occurrence of a steady free-molecular flow with higher accuracy. Then the latter is performed for more diverse values of $\alpha_{n}$ and $\alpha_{t}$ and also for finite $\mathrm{Kn}$ to examine the transition of the flow field depending on those parameters. In the case of the Lord model (8), we only carry out the computation for the free-molecular gas by the DSMC method.

In the following, we will show the results for the CL model in Sec. III A and those for the Lord model in Sec. III B. A brief description of the computation of the integral equation (15) will be given in Appendix A, whereas that of the DSMC method will be omitted because we simply follow the standard procedure explained in Appendix B.1 in Ref. 4 (see also Refs. 32 and 35 for incorporation of the boundary condition into the DSMC). Data concerning the computational condition (such as the number of cells or simulation particles in the DSMC, etc.) and the numerical error will also be given in Appendix A.

\section{A. Results for the $\mathrm{CL}$ model}

\section{Behavior of the free-molecular gas $(\mathrm{Kn} \rightarrow \infty)$}

Figures 1-5 show typical behavior of the free-molecular gas in the case of the CL model with $\left(\alpha_{n}, \alpha_{t}\right)=(0.5,0.5)$, $(0.7,0.5),(1,0.25),(1,1.75)$, and $(1,1)$ obtained by computations based on the integral equation (15). Recall that the back scattering is dominant for $\alpha_{t}>1$.

The temperature of the boundary (at $x_{2}=0.5$ ) takes its maximum $\hat{T}_{w}=1.5$ at $x_{1}=0.5$ and minimum $\hat{T}_{w}=0.5$ at $x_{1}=$ -0.5 . Accordingly, the gas temperature has a gradient in the rightward (positive $x_{1}$ ) direction on each cross section $x_{2}$ $=$ const., which is steepest on the plate and becomes more gradual as the centerline $x_{2}=0$ is approached [see panel (a) of Figs. 1-5]. The temperature of the gas near the plate is best accommodated to that of the plate in Fig. 5(a), i.e., in the case of $\left(\alpha_{n}, \alpha_{t}\right)=(1,1)$ corresponding to the diffuse reflection.

The density distributions in Figs. 1(b), 2(b), and 5(b) are dissimilar to those in Figs. 3(b) and 4(b). In the former fig-

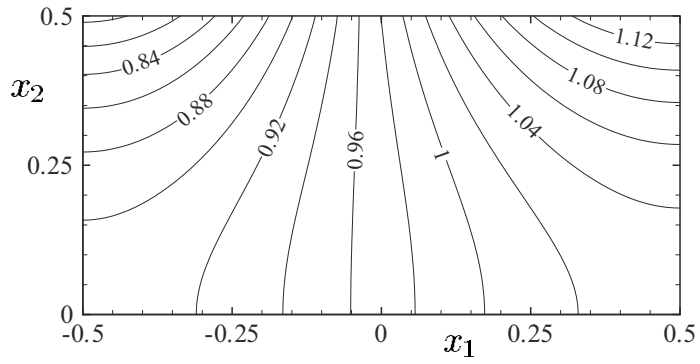

(a) Isolines of $\hat{T}$

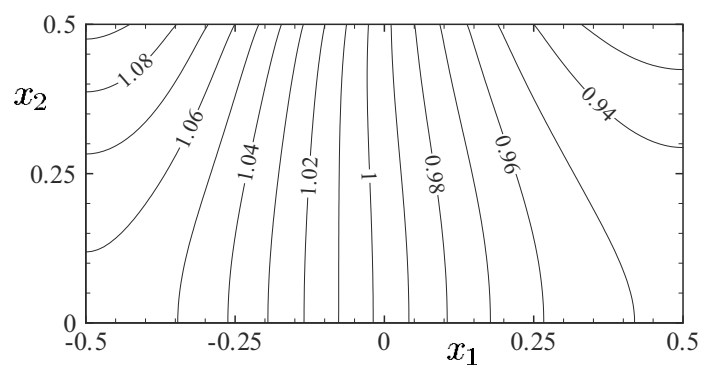

(b) Isolines of $\hat{\rho}$

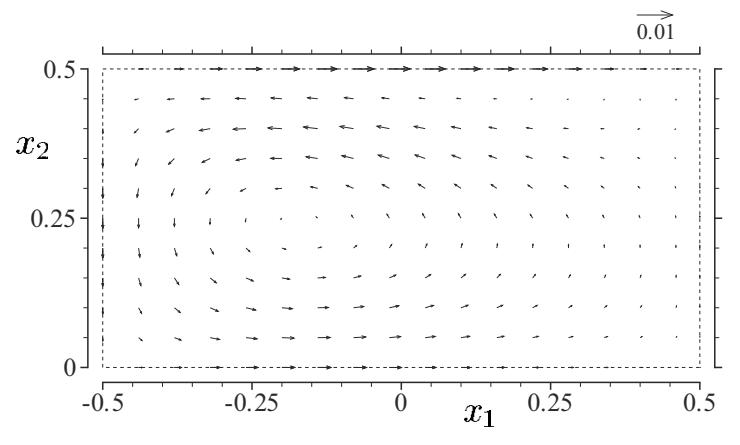

(c) 2-D vector $\left(u_{1}, u_{2}\right)$

FIG. 1. Behavior of the free-molecular gas $(\mathrm{Kn} \rightarrow \infty)$ in the case of the Cercignani-Lampis model with $\left(\alpha_{n}, \alpha_{t}\right)=(0.5,0.5)$. (a) Isolines of the (dimensionless) temperature $\hat{T}$, (b) those of the density $\hat{\rho}$, and (c) the flow velocity $\left(u_{1}, u_{2}\right)$.

ures, the density gradient along $x_{2}=$ const. is in the leftward (negative $x_{1}$ ) direction, which is opposite to the direction of the temperature gradient. In the latter figures, the density gradient along $x_{2}=$ const. changes its direction in the gas because of the presence of a saddle point. In this way, the patterns of the isodensity lines exhibit larger variations than the patterns of the isothermal lines depending on the accommodation coefficients.

As seen in panel (c) of Figs. 1-4, steady free-molecular flows are induced by the effect of nonuniform temperature of the boundary, contrary to the case of $\left(\alpha_{n}, \alpha_{t}\right)=(1,1)$ corresponding to the diffuse reflection (i.e., Fig. 5). Note that the magnitude of the reference vector is different in each figure. The pattern of the flow field varies according to the accommodation coefficients and may be classified into four types illustrated in the above four figures. In Figs. 1(c) and 2(c), the flow field is dominated by a vortex rotating counterclockwise around $\left(x_{1}, x_{2}\right)=(-0.15,0.25)$. The gas on the plate (at $x_{2}=0.5$ ) flows rightward (from colder part to hotter part) in Fig. 1(c), whereas a leftward flow is dominant in Fig. 2(c). In 


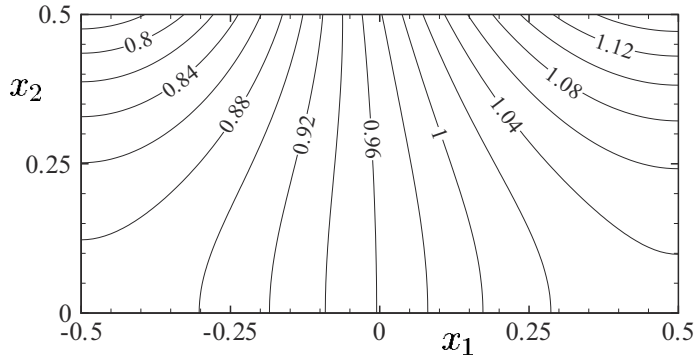

(a) Isolines of $\hat{T}$

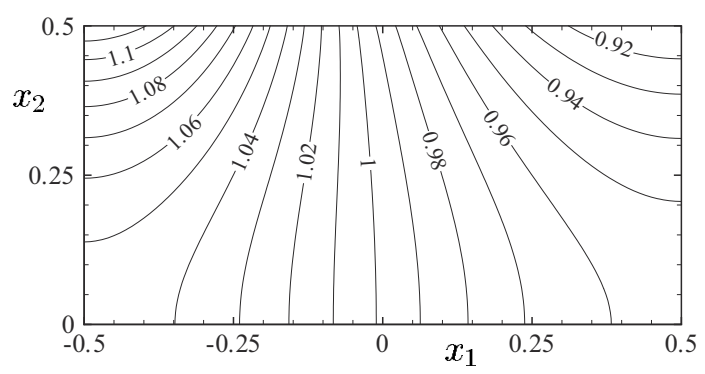

(b) Isolines of $\hat{\rho}$

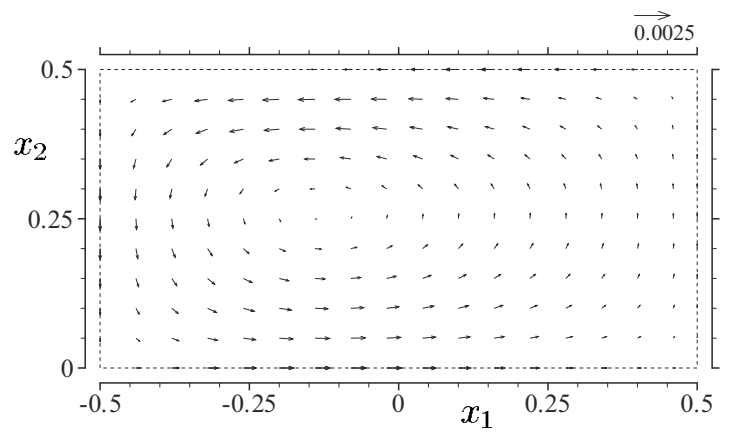

(c) 2-D vector $\left(u_{1}, u_{2}\right)$

FIG. 2. Behavior of the free-molecular gas $(\mathrm{Kn} \rightarrow \infty)$ in the case of the Cercignani-Lampis model with $\left(\alpha_{n}, \alpha_{t}\right)=(0.7,0.5)$. See the caption of Fig. 1.

the former figure, the direction of the flow on the plate is opposite to that induced by the central vortex, so that there exists a thin clockwise vortex in the neighborhood of the plate. The patterns of the flow field seen in Figs. 3(c) and 4(c) are just the reverse of those in Figs. 1(c) and 2(c). That is, the flow field is dominated by a clockwise vortex and the gas on the plate flows leftward (from hotter part to colder part) in Fig. 3(c) and flows rightward in Fig. 4(c). In the following, we shall call the above four types of the flow field illustrated in Figs. 1(c), 2(c), 3(c), and 4(c) the I-, II-, III-, and IV-types, respectively, i.e.,

- I-type: A dominant counterclockwise vortex in the gas and a dominant rightward flow on the plate.

- II-type: A dominant counterclockwise vortex in the gas and a dominant leftward flow on the plate.

- III-type: A dominant clockwise vortex in the gas and a dominant leftward flow on the plate.

- IV-type: A dominant clockwise vortex in the gas and a dominant rightward flow on the plate.

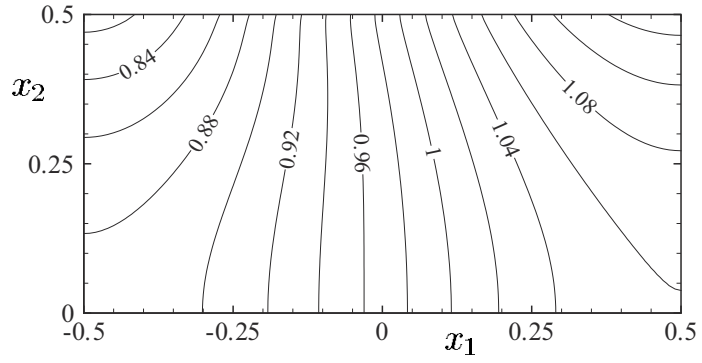

(a) Isolines of $\hat{T}$

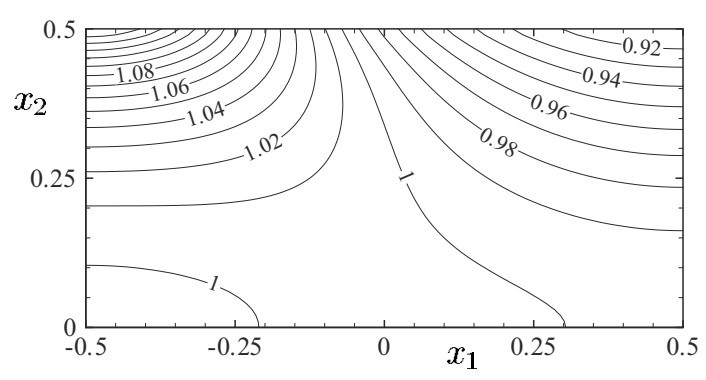

(b) Isolines of $\hat{\rho}$

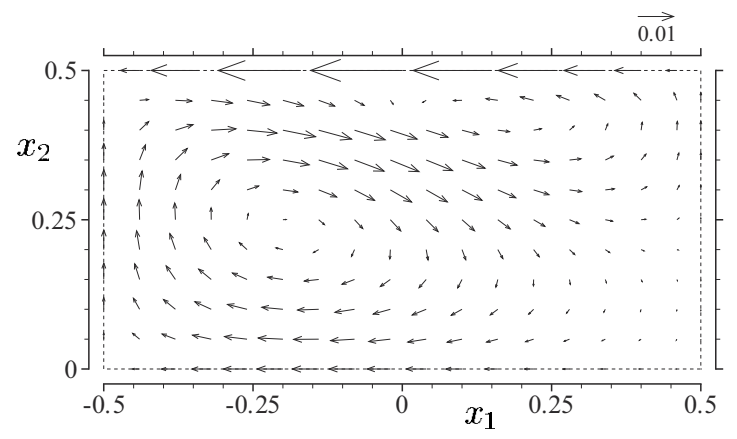

(c) 2-D vector $\left(u_{1}, u_{2}\right)$

FIG. 3. Behavior of the free-molecular gas $(\mathrm{Kn} \rightarrow \infty)$ in the case of the Cercignani-Lampis model with $\left(\alpha_{n}, \alpha_{t}\right)=(1,0.25)$. See the caption of Fig. 1.

By the way, the CL model was compared with the data of molecular beam scattering experiments conducted for smooth metal surfaces and noble gases and appropriate values of the accommodation coefficients were determined in Ref. 23. All the values of the accommodation coefficient $\alpha_{t}$ for tangential momentum reported in the above reference, which differ depending on surface materials, gases, and temperature, are less than unity $\left(0.06 \lesssim \alpha_{t} \leqslant 0.9\right)$. Physical intuition as well as the result of the above reference may suggest that $\alpha_{t}$ would be less than unity for ordinary smooth surfaces and a sort of rough surface should be considered to realize the situation where $\alpha_{t}>1$ (or the back scattering is dominant; see the third paragraph in Sec. II B). Incidentally, the CL model with $\alpha_{t} \simeq 1.045$ was found to approximate well some features of the conical rough surface model (with $66^{\circ}$ opening angle) in Ref. 36 (a similar example was reported also in Ref. 37). To the best of the authors' knowledge, however, no example of a surface whose $\alpha_{t}$ is well over unity as in Fig. 4 has been reported in the literature so far. This point should be kept in mind also in Sec. III A 2. 


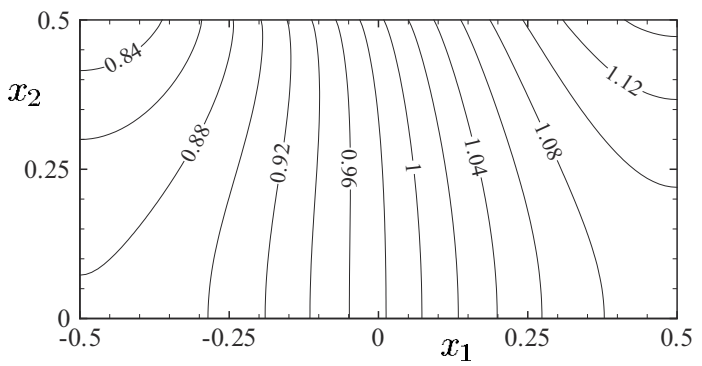

(a) Isolines of $\hat{T}$

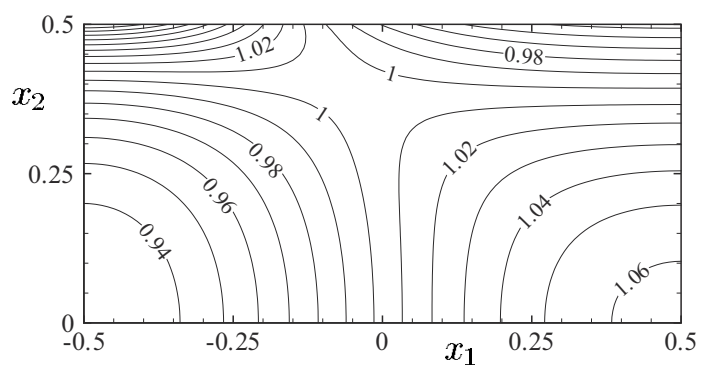

(b) Isolines of $\hat{\rho}$

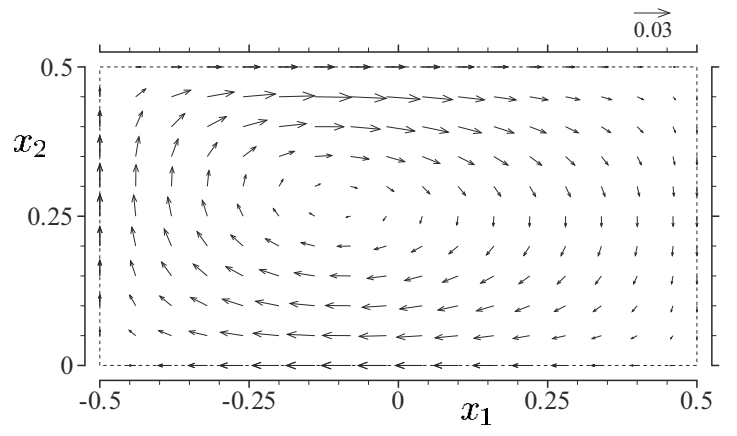

(c) 2-D vector $\left(u_{1}, u_{2}\right)$

FIG. 4. Behavior of the free-molecular gas $(\mathrm{Kn} \rightarrow \infty)$ in the case of the Cercignani-Lampis model with $\left(\alpha_{n}, \alpha_{t}\right)=(1,1.75)$. See the caption of Fig. 1.

\section{Transition of the flow field}

Here, we carry out the DSMC computations changing the Knudsen number as well as the accommodation coefficients and classify the results into the four types introduced in Sec. III A 1 according to the pattern of the flow field. The classification is implemented objectively on the basis of quantitative criteria, the detail of which will be given in Appendix A. Figure 6 shows the results of the classification, where the size of symbols indicates the strength of the flow (i.e., the maximum flow speed).

The classification in the case of the free-molecular gas is shown in panel (a) of Fig. 6 [i.e., panel (a) includes the cases corresponding to Figs. 1-5]. The solid line in the figure indicates a parabola defined by $\alpha_{n}=\alpha_{t}\left(2-\alpha_{t}\right)$. The figure shows a strong correlation between which of $\alpha_{n}$ and $\alpha_{t}(2$ $-\alpha_{t}$ ) is larger and in which direction the main vortex in the flow field rotates. Except for a few points near the parabola, points under the parabola [i.e., $\alpha_{n}<\alpha_{t}\left(2-\alpha_{t}\right)$ ] are classified into the I- or II-type, each of which contains a dominant counterclockwise vortex in the flow field. On the other hand, points above the parabola [i.e., $\left.\alpha_{n}>\alpha_{t}\left(2-\alpha_{t}\right)\right]$ are classified

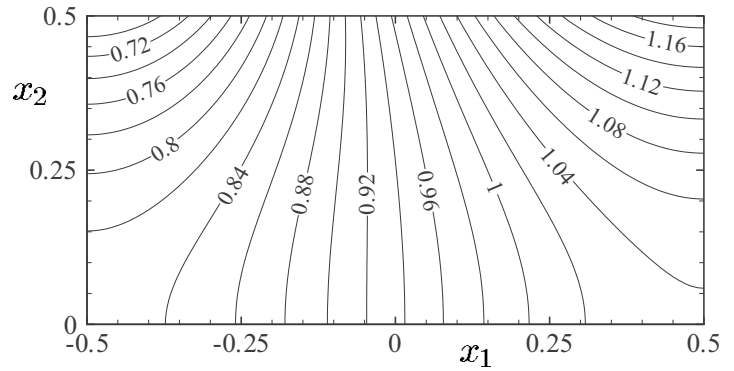

(a) Isolines of $\hat{T}$

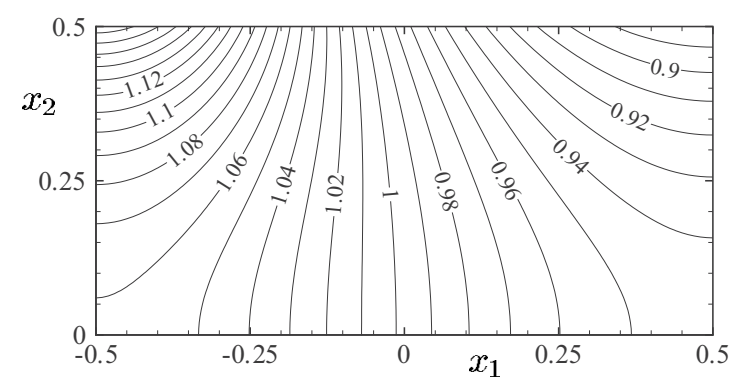

(b) Isolines of $\hat{\rho}$

FIG. 5. Behavior of the free-molecular gas $(\mathrm{Kn} \rightarrow \infty)$ in the case of the Cercignani-Lampis model with $\left(\alpha_{n}, \alpha_{t}\right)=(1,1)$, which is equivalent to the diffuse reflection. (a) Isolines of the (dimensionless) temperature $\hat{T}$ and (b) those of the density $\hat{\rho}$.

into the III-type in the case of $\alpha_{t}<1$ or the IV-type in the case of $\alpha_{t}>1$ and the dominant vortex rotates in the clockwise direction. As mentioned in the third paragraph in Sec. II B, $\alpha_{n}$ is the accommodation coefficient for partial kinetic energy associated with the normal velocity component $\zeta_{2}$ and $\alpha_{t}\left(2-\alpha_{t}\right)$ is that associated with the tangential velocity components $\zeta_{1}$ and $\zeta_{3}$. If $\alpha_{n}>\alpha_{t}\left(2-\alpha_{t}\right)$, therefore, the distribution of $\zeta_{2}$ of reflected molecules on the plate is accommodated to the plate's temperature better than the distribution of $\zeta_{1}$ and $\zeta_{3}$ and vice versa. Another important feature of Fig. 6 (a) is that the symbols become larger with the increase of the distance from the parabola, irrespective of the type of the flow field. This means that faster flows are induced as the velocity distribution of reflected molecules on the plate becomes more anisotropic (with growing discrepancy between the distribution of $\zeta_{2}$ and that of $\zeta_{1}$ and $\zeta_{3}$ ) or, in other words, as the boundary condition becomes more non-Lambertian (see the next-to-last paragraph in Sec. I). However, it should be noted that even at a point on the parabola [except $\left.\left(\alpha_{n}, \alpha_{t}\right)=(1,1)\right]$, the CL model is still non-Lambertian and the flow is induced in general as shown in the next Sec. III B.

The effect of intermolecular collisions on the flow pattern is shown in panels (b) and (c) of Fig. 6. Contrary to the case of the free-molecular gas, flows are induced also at $\left(\alpha_{n}, \alpha_{t}\right)=(1,1)$. The classification in the case of $\mathrm{Kn}=1$ [Fig. 6(b)], where the reference mean free path is equal to the gap between the plates, is almost the same as that for the freemolecular flow [Fig. 6(a)]. Conversely, it seems that the flow pattern of the free-molecular gas in Fig. 6(a) is determined mainly by the contribution of molecules coming directly from the neighboring area on the plates (within a distance 


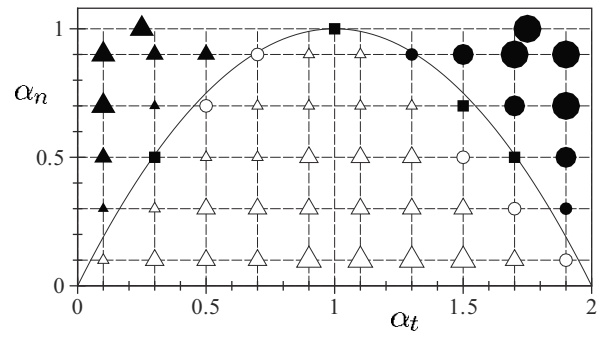

(a) $\mathrm{Kn} \rightarrow \infty$

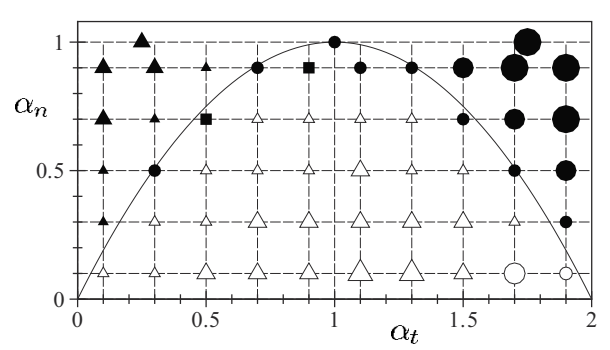

(b) $\mathrm{Kn}=1$

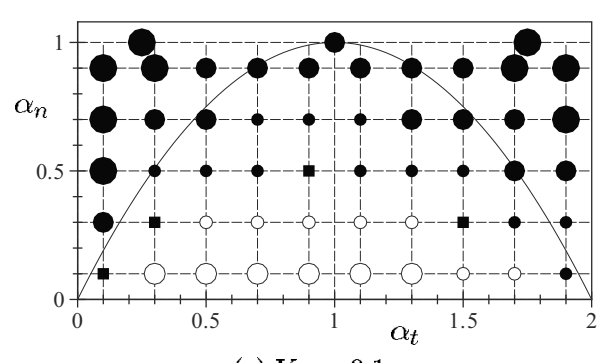

(c) $\mathrm{Kn}=0.1$

FIG. 6. Type of the flow field in the case of the Cercignani-Lampis model with various $\alpha_{n}$ and $\alpha_{t}$. (a) $\mathrm{Kn} \rightarrow \infty$, (b) $\mathrm{Kn}=1$, and (c) $\mathrm{Kn}=0.1$. Here, $\triangle, \bigcirc$, $\boldsymbol{\Delta}$, and - indicate the I-, II-, III-, and IV-type flows, respectively. The symbol size indicates the maximum flow speed $U\left[\equiv \max \left(u_{1}^{2}+u_{2}^{2}\right)^{1 / 2}\right]$; small symbols are used for $1 \times 10^{-3} \leq U<8 \times 10^{-3}$, middle symbols for $8 \times 10^{-3}$ $\leq U<1.6 \times 10^{-2}$, and large symbols for $U \geq 1.6 \times 10^{-2}$. $\boldsymbol{\square}$ indicates the case where $U<1 \times 10^{-3}$ or the flow field is not classified into any of the four types. The solid line is the parabola $\alpha_{n}=\alpha_{t}\left(2-\alpha_{t}\right)$.

comparable to the gap between the plates) and is less affected by the far field. However, the classification in the case of $\mathrm{Kn}=0.1$ [Fig. 6(c)] is quite different. That is, only the IIand IV-types are seen in the small- and large- $\alpha_{n}$ region, respectively. The I- and III-types, which involve, in the neighborhood of the plate, a strong shear due to the discrepancy between the flow direction on the plate and the direction of the main vortex, disappear because of an increasing effect of molecular collisions. Figure 7 shows the transition of flow field for $\left(\alpha_{n}, \alpha_{t}\right)=(0.5,0.5)$ with decreasing $\mathrm{Kn}$. The flow field for $\mathrm{Kn}=1$ is classified into the I-type, which is the same as in the free-molecular gas [see Fig. 1(c)], while that for $\mathrm{Kn}=0.1$ exhibits the central vortex in the opposite direction and thus belongs to the IV-type.

\section{B. Result for the Lord model and extension to general Lambertian conditions}

As indicated by Fig. 6(a) in Sec. III A 2, the main cause of the free-molecular flow seems to be the anisotropic velocity distribution of reflected molecules produced by the nonLambertian boundary condition. In this section, we consider

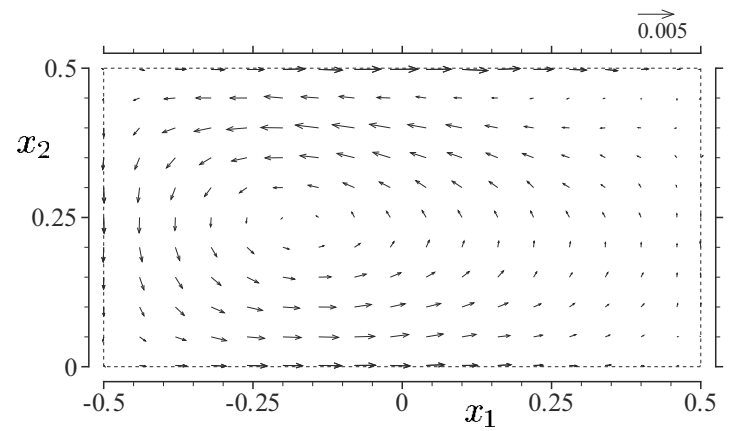

(a) $\mathrm{Kn}=1$

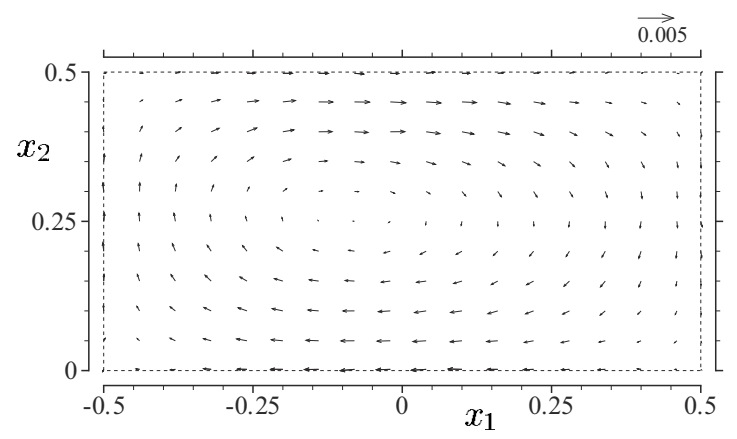

(b) $\mathrm{Kn}=0.1$

FIG. 7. Flow velocity $\left(u_{1}, u_{2}\right)$ in the case of the Cercignani-Lampis model with $\left(\alpha_{n}, \alpha_{t}\right)=(0.5,0.5)$. (a) $\mathrm{Kn}=1$ and (b) $\mathrm{Kn}=0.1$.

the case of a Lambertian boundary condition: first, numerical results for the Lord model (the diffuse reflection with incomplete energy accommodation) will be compared to those of the CL model and then, a more general case will be considered.

\section{Numerical results for the Lord model}

The behavior of the free-molecular gas in the case of the CL model with $\left(\alpha_{n}, \alpha_{t}\right)=(0.75,0.5)$ and that in the case of the Lord model with $\sigma=0.75$ are compared in Fig. 8, where the former was obtained by the computation of the integral equation (15) and the latter by the DSMC method. The total energy accommodation coefficient $(=0.75)$ is common to both models (see the fifth paragraph in Sec. II B). The former model is non-Lambertian, though the anisotropy in the velocity distribution of reflected molecules is relatively weak since the accommodation coefficients are taken at a point on the parabola in Fig. 6(a). On the other hand, the latter model is Lambertian. As shown in panels (a) and (b), both models yield quite similar temperature and density fields. However, the flow field is different: a relatively weak flow shown in panel (c) is induced in the case of the CL model, whereas no flow is induced in the case of the Lord model.

\section{General Lambertian conditions}

The above result, i.e., the absence of a steady freemolecular flow in the case of the Lord model, can be understood as one specific example of the following general consideration. Let us consider a free-molecular gas around one or several bodies at rest which have arbitrary shapes and distributions of surface temperature. The configuration and 


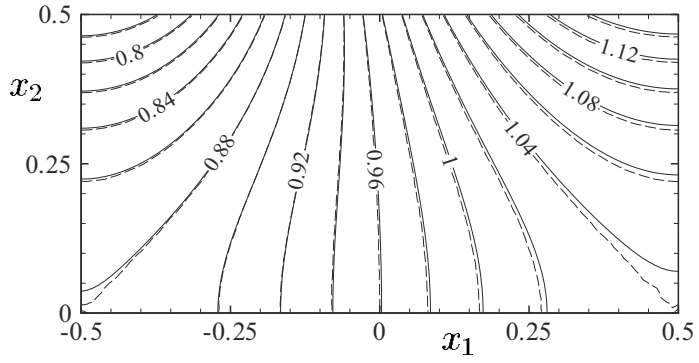

(a) Isolines of $\hat{T}$

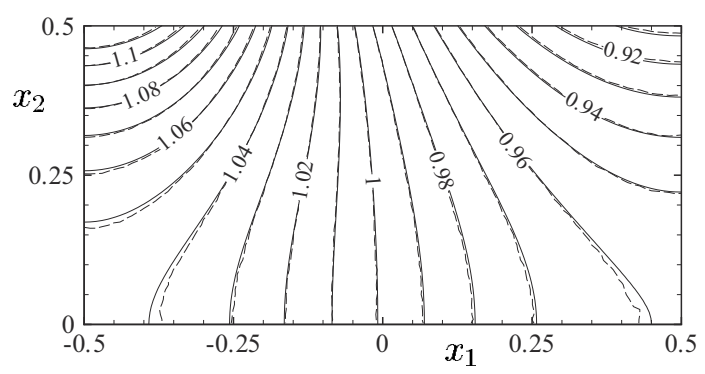

(b) Isolines of $\hat{\rho}$

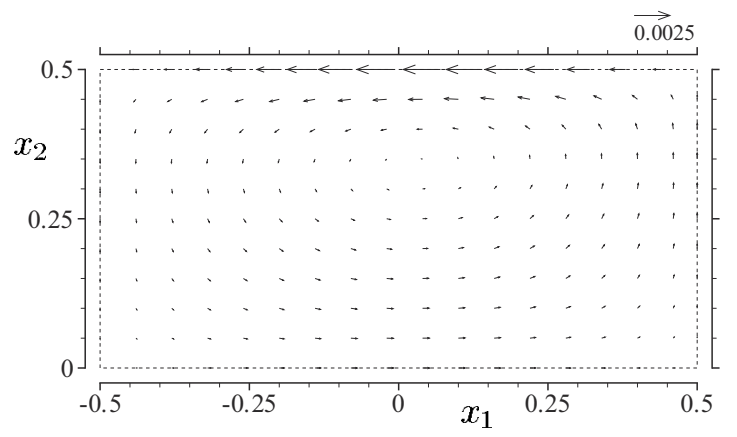

(c) 2-D vector $\left(u_{1}, u_{2}\right)$

FIG. 8. Comparison of behavior of the free-molecular gas $(\mathrm{Kn} \rightarrow \infty)$ under the different boundary conditions. (a) Isolines of the (dimensionless) temperature $\hat{T}$, (b) those of the density $\hat{\rho}$, and (c) the flow velocity $\left(u_{1}, u_{2}\right)$. In panels (a) and (b), the solid lines indicate results of the Cercignani-Lampis model with $\left(\alpha_{n}, \alpha_{t}\right)=(0.75,0.5)$ and the dashed lines indicate those of the Lord model with $\sigma=0.75$. Panel (c) shows the results for the CercignaniLampis model.

number of the bodies are also arbitrary. In this subsection, we use the same notations as in the previous sections. However, they should be interpreted appropriately. For instance, $L$ should be the characteristic length of the system, $T_{0}$ should be the reference temperature, $L \boldsymbol{x}$ should be the position vector in the three-dimensional physical space, $T_{0} \hat{T}_{w}$ should be the temperature of the surfaces of the bodies depending on the position $\boldsymbol{x}$, etc.

For the moment, we assume that the gas and the bodies are confined in a large closed vessel. Furthermore, we assume a Lambertian boundary condition on surfaces of the bodies and vessel expressed as follows. For $\boldsymbol{x} \in S$ and $\boldsymbol{\zeta} \cdot \boldsymbol{n}$ $>0$,

$$
f(\boldsymbol{x}, \boldsymbol{\zeta})=\int_{\zeta_{*} \cdot \boldsymbol{n}<0}\left|\boldsymbol{\zeta}_{*} \cdot \boldsymbol{n}\right| K\left(\zeta_{*}, \zeta ; \boldsymbol{x}\right) f\left(\boldsymbol{x}, \boldsymbol{\zeta}_{*}\right) \mathrm{d} \boldsymbol{\zeta}_{*}
$$

where $S$ denotes the surfaces and $\boldsymbol{n}$ denotes the unit normal vector at the point $\boldsymbol{x} \in S$ pointing to the gas. It should be noted that the arguments $\zeta_{*}$ and $\zeta$ of the kernel $K$ are the absolute values of $\zeta_{*}$ and $\zeta$, respectively [see Eq. (8b)]. Thus, the kernel $K$ is independent of the direction of $\zeta$ and so is $f$ in the left-hand side (LHS) of Eq. (17). It is well-known that any scattering kernel for ordinary nonabsorbing surfaces should satisfy three fundamental properties, i.e., (i) nonnegativity, (ii) normality, and (iii) reciprocity (see, e.g., Ref. 1). These properties may be expressed as the following relations for $K$ :

$$
\begin{aligned}
& K\left(\zeta_{*}, \zeta ; \boldsymbol{x}\right) \geq 0, \\
& \pi \int_{0}^{\infty} \zeta^{3} K\left(\zeta_{*}, \zeta ; \boldsymbol{x}\right) \mathrm{d} \zeta=1, \\
& \exp \left(-\frac{\zeta_{*}^{2}}{\hat{T}_{w}}\right) K\left(\zeta_{*}, \zeta ; \boldsymbol{x}\right)=\exp \left(-\frac{\zeta^{2}}{\hat{T}_{w}}\right) K\left(\zeta, \zeta_{*} ; \boldsymbol{x}\right) .
\end{aligned}
$$

Now let us introduce a unit vector $\ell \equiv \zeta / \zeta$ and define the marginal distribution function $\hat{g}$ as

$$
\hat{g}(\boldsymbol{x}, \ell)=\int_{0}^{\infty} \zeta^{3} f(\boldsymbol{x}, \zeta \ell) \mathrm{d} \zeta .
$$

Since molecular collisions are absent, the Boltzmann equation for $\hat{g}$ is written simply as

$$
\ell \cdot \frac{\partial \hat{g}}{\partial \boldsymbol{x}}=0
$$

The boundary condition for $\hat{g}$ is derived from Eqs. (17) and (18b). For $\boldsymbol{x} \in S$ and $\ell \cdot \boldsymbol{n}>0$,

$$
\hat{g}(\boldsymbol{x}, \ell)=\frac{1}{\pi} \int_{\ell_{*} \cdot \boldsymbol{n}<0}\left|\ell_{*} \cdot \boldsymbol{n}\right| \hat{g}\left(\boldsymbol{x}, \ell_{*}\right) \mathrm{d} \Omega\left(\ell_{*}\right),
$$

with $\ell_{*}$ being a unit vector. Here we should note that $\hat{g}$ in the LHS of Eq. (21) is, in fact, independent of $\ell$ and also of surface properties, such as temperature and accommodation coefficients, because the kernel $K$ has been eliminated by the use of Eq. (18b). Therefore, it is immediately seen that $\hat{g}$ $=$ const. can be a solution for the boundary-value problem, i.e., Eqs. (20) and (21). The flow velocity corresponding to such a constant solution is given by

$$
\hat{\rho} \boldsymbol{u}=\int \zeta \mathrm{d} \mathrm{\zeta}=\hat{g} \int \ell \mathrm{d} \Omega(\ell)=0 .
$$

The conclusion is that no steady free-molecular flow is induced around the bodies irrespective of the distribution of surface temperature and accommodation coefficients, when the boundary condition can be written in the form of Eq. (17) and the kernel $K$ satisfies Eq. (18). The Lord model is included in this case.

The above discussion may be extended to the case of an infinite domain if the gas at infinity is in a state which gives the same constant $\hat{g}$ [e.g., $f=C \beta^{2} \exp \left(-\beta \zeta^{2}\right)$ in Ref. 15]. In the case of a closed domain, uniqueness of the solution for the boundary-value problem, i.e., Eqs. (20) and (21), (up to a 
multiplicative constant) can be verified through essentially the same argument as in Ref. 15 under the same assumptions: (i) any two points on $S$ can be connected by a chain of straight lines (or a zigzag line) passing through the gas whose joints are on $S$ and (ii) $\hat{g}(\boldsymbol{x}, \ell)$ for $\boldsymbol{x} \in S$ and $\ell \cdot \boldsymbol{n}>0$ is non-negative and continuous on $S$. Furthermore, the absence of the flow can be verified also for the boundary condition of the form of a linear combination of the Lambertian and the specular-reflection condition [i.e., the Maxwell-type model with its diffuse-reflection part being replaced by the condition expressed in Eq. (17)] under the assumption that the gas at infinity gives $\hat{g}=$ const. in the case of an infinite domain (see Appendix B).

\section{CONCLUDING REMARKS}

In the present study, we have considered a rarefied gas between two parallel plates with sinusoidal temperature distribution. We have investigated the steady behavior of the gas on the basis of the Boltzmann equation and the CL model or the Lord model (diffuse reflection with incomplete energy accommodation) as a boundary condition on the plates, with special interest in the free-molecular limit.

For the CL model, an accurate deterministic computation based on the integral equation for the free-molecular gas has been performed. The result demonstrates clearly that a steady flow is induced by the nonuniform temperature distribution of the plates even in the free-molecular limit. This is in contrast to the fact that such a flow vanishes in the freemolecular limit for the Maxwell-type model. In addition, the DSMC computation has been performed for wide range of the accommodation coefficients to illustrate the transition of the flow pattern among the four typical types defined in the present study. It is particularly interesting that the dominant vortex in the flow field rotates counterclockwise in the region $\alpha_{n} \leqslant \alpha_{t}\left(2-\alpha_{t}\right)$ and clockwise in the region $\alpha_{n} \geqslant \alpha_{t}\left(2-\alpha_{t}\right)$. The results also indicate correlation between the strength of the induced free-molecular flow and the degree of anisotropy in the velocity distribution of molecules reflected from the boundary.

We have also carried out the DSMC computation for finite Knudsen numbers in order to see the effect of intermolecular collisions on the flow field. The result shows that the flow pattern for $\mathrm{Kn}=1$ is roughly the same as that for the free-molecular limit, whereas that for $\mathrm{Kn}=0.1$ is quite dissimilar.

Furthermore, the behavior of the free-molecular gas has been investigated for the Lord model and the result has been compared with the corresponding result for the CL model with the common energy accommodation coefficient. The temperature and density fields obtained by the two models are quite similar to each other. However, no steady flow is induced for the Lord model, as in the case of the Maxwelltype model.

Finally, we have considered, as a generalization of the Lord model, the boundary condition that gives the cosine law (Lambert's law) for the reflected molecular flux in its general form. We have investigated the steady behavior of a freemolecular gas under such a boundary condition in an arbi- trary configuration and showed that the flow due to a steady temperature field vanishes. Then it was shown that the same is true for the boundary condition consisting of the general Lambertian condition and the specular reflection.

\section{ACKNOWLEDGMENTS}

This work was completed while two of the authors (K.A. and S.T.) were staying at the Issac Newton Institute for Mathematical Sciences. They thank the Institute for its hospitality and support. This work was partially supported by the Grants-in-Aid for Scientific Research, No. 21760129 from MEXT and No. 20360046 from JSPS.

K.A. wishes to express his heartfelt thanks to the late Professor Carlo Cercignani for his guidance, collaboration, and friendship over the last three decades.

\section{APPENDIX A: DATA FOR THE COMPUTATIONS}

\section{Computation of the integral equation for the free- molecular gas in the case of the CL model}

We first explain the numerical computation of the integral equation (15) briefly. In the actual computation, the following $z$ and $s$ instead of $\zeta_{1}$ and $\zeta_{2}$ were introduced as independent variables:

$$
z=\left(\zeta_{1}^{2}+\zeta_{2}^{2}\right)^{1 / 2}, \quad s=\zeta_{1} /\left|\zeta_{2}\right|
$$

They were restricted within finite intervals, i.e., $0 \leq z \leq z_{\max }$ and $-N \lambda \leq s \leq N \lambda$. We used uniform grids for all the independent variables: $x_{1}^{(i)}\left(i=-M, \ldots, 0, \ldots, M ; x_{1}^{(0)}=0, x_{1}^{( \pm M)}\right.$ $= \pm \lambda / 4), \quad z^{(j)} \quad\left(j=0, \ldots, Z ; \quad z^{(0)}=0, \quad z^{(Z)}=z_{\max }\right), \quad$ and $s^{(k)}$ $\left(k=-4 N M, \ldots, 0, \ldots, 4 N M ; s^{(0)}=0, s^{( \pm 4 N M)}= \pm N \lambda\right)$. The solution $g$ and $h$ of Eq. (15) was obtained by the method of successive approximations starting with appropriate initial distribution; Eq. (15a) was solved first and the resulting $g$ was then substituted into the last term on RHS of Eq. (15b). To obtain a unique solution of Eq. (15a), we adjusted $g$ by multiplying a constant at the end of each iteration step so that the total amount of the gas involved was fixed during the computation (see the second paragraph in Sec. II C). The numerical integration with respect to $z$ and $s$ was carried out by Simpson's rule. Since the grid size for $x_{1}$ and that for $s$ are equal, Simpson's rule for Eq. (16) can be carried out by referring only to $g$ or $h$ at the grid points. On the other hand, values of $g$ and $h$ at points not on the grids are necessary, in general, to perform Simpson's rule for Eq. (14) for the macroscopic quantities at arbitrary $\left(x_{1}, x_{2}\right)$. We used the thirddegree Lagrange polynomial for interpolation in such a case. 
We prepared the following four grid systems:

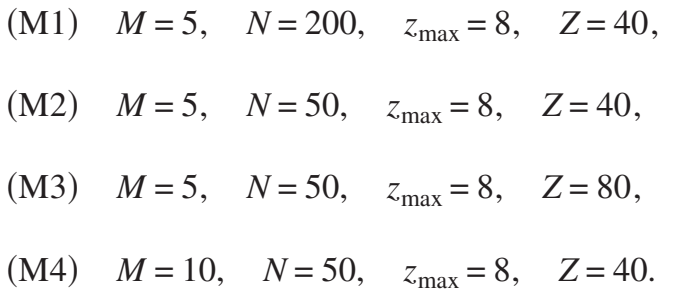

All the results shown in Figs. 1-5 and 8 were obtained with the grid (M1). The number of $x_{1}$ grids $M$ may seem to be small, but it was sufficient in the present analysis because $g$ and $h$ vary smoothly as a function of $x_{1}$ in accordance with the sinusoidal temperature of the plate. Rather than $M$, the number of $s$ grids $N$ had more influence on numerical error in some cases. In the case of $\left(\alpha_{n}, \alpha_{t}\right)=(1,1)$, the maximum flow speed $U\left[\equiv \max \left(u_{1}^{2}+u_{2}^{2}\right)^{1 / 2}\right]$, which is theoretically zero in this case, can be a measure of numerical error: $U \simeq 1.2$ $\times 10^{-9}$ in the (M1) system and $U \lesssim 2.2 \times 10^{-9}$ in the other systems. For other pairs of $\left(\alpha_{n}, \alpha_{t}\right)$, the maximum value of $\left|u_{2}\right|$ on the plate $\left(x_{2}=0.5\right)$, which is also theoretically zero, can be used as a measure of the error: $\left|u_{2}\left(x_{2}=0.5\right)\right| \leq 4.6$ $\times 10^{-7}$ for $\left(\alpha_{n}, \alpha_{t}\right)=(0.5,0.5),(1,0.25)$, and $(1,1.75)$, and $\left|u_{2}\left(x_{2}=0.5\right)\right| \leqslant 2.0 \times 10^{-8} \quad$ for $\quad\left(\alpha_{n}, \alpha_{t}\right)=(0.7,0.5) \quad$ and $(0.75,0.5)$ in the (M1) system; and $\left|u_{2}\left(x_{2}=0.5\right)\right| \lesssim 6.9 \times 10^{-6}$ for $\left(\alpha_{n}, \alpha_{t}\right)=(0.5,0.5)$ and $(1,0.25)$ in the other systems. The difference between the density or temperature distribution for $\left(\alpha_{n}, \alpha_{t}\right)=(0.5,0.5),(1,0.25)$, and $(1,1)$ obtained in the (M1) system and that obtained in the other systems is less than $0.2 \%$.

\section{Computation by the DSMC method}

All the results of the DSMC shown in Sec. III (i.e., Figs. 6 and 7 and the result of the Lord model in Fig. 8) were obtained under the same computational conditions. We prepared $201 \times 61$ rectangular cells along the $x_{1}$ and $x_{2}$ directions in an extended domain $\left|x_{1}\right| \leq \lambda / 2$ (instead of $\left|x_{1}\right|$ $\leq \lambda / 4)$ and $0 \leq x_{2} \leq 1 / 2$, and imposed the periodic boundary condition at $x_{1}= \pm \lambda / 2$ instead of the specular-reflection condition at $x_{1}= \pm \lambda / 4$ (see the last paragraph in Sec. II B). The cells were uniform in size except on the edge of the computational domain: cells adjacent to the boundaries were half the size of the standard cell (cells in the four corners were quarter the size). The number of simulation particles was 4 $\times 10^{6}$ (about 333 per standard cell) and the time step was $0.001 t_{0}\left[t_{0} \equiv L /\left(2 k T_{0} / m\right)^{1 / 2}\right]$. After the steady state was established, we took the time average of 20000 snapshots taken at every 10 steps (i.e., at intervals of $0.01 t_{0}$ ). The results obtained in the extended domain satisfy the mirror symmetry with respect to $x_{1}= \pm \lambda / 4$ [see Eqs. (9b) and (9c)] within the computational accuracy. For example, the flow velocities $u_{1}$ and $u_{2}$ satisfy the mirror symmetry within an average error of $7.4 \times 10^{-4}$ (average taken over the cells). Thus, to obtain smoother final results in $\left|x_{1}\right| \leq \lambda / 4$, we took the average of macroscopic quantities in two cells located at the symmetrical positions.

In the case of the free-molecular gas, we sometimes took the ensemble average of ten independent trials to further re- duce the statistical fluctuation. To be more specific, we took the ensemble average in the representative cases as shown in Figs. 1-5 or in the case of weak flow where the average flow speed $\bar{u}$ [the average of $\left(u_{1}^{2}+u_{2}^{2}\right)^{1 / 2}$ over all the cells] was less than $1 \times 10^{-3}$. The $\bar{u}$ for the CL model with $\left(\alpha_{n}, \alpha_{t}\right)=(1,1)$ and for the Lord model, which are zero theoretically, give a measure of the fluctuation: $\bar{u} \leqslant 2.5 \times 10^{-4}$ in a single trial and $\$ 7.9 \times 10^{-5}$ in the ensemble average of ten trials. The flow velocity $u_{3}$ along the $x_{3}$ direction, which also should be zero, can be another measure of the fluctuation [for arbitrary $\left(\alpha_{n}, \alpha_{t}\right)$ ]: the average $\bar{u}_{3}$ (taken over all the cells) is less than $2.7 \times 10^{-4}$ in a single trial and less than $7.3 \times 10^{-5}$ in the ensemble average of ten trials. In the case of $\mathrm{Kn}=1$ or 0.1 , the ensemble average was taken only in a few representative cases including the case shown in Fig. 7.

In the classification of the flow field shown in Fig. 6, we first computed the following two quantities: (i) the circulation of the two-dimensional vector $\left(u_{1}, u_{2}\right)$ along the rectangular closed path $\left[\left(x_{1}, x_{2}\right)=\right] \quad(0.4,0.4)-(0.4,0.1)$ $(-0.4,0.1)-(-0.4,0.4)-(0.4,0.4)$ and (ii) the line integral of $u_{1}$ along the line $(-0.4,0.497917)-(0.4,0.497917)$ near the plate. Here we denote (i) and (ii) divided by the length of each integration path as $J_{1}$ and $J_{2}$, respectively. If $J_{1}>0$, the flow field contains a dominant vortex rotating in the clockwise direction; if $J_{2}>0$, the gas on the plate flows toward the right (the direction from the colder part to the hotter part). Then, the flow field was classified into the I-type if $J_{1}<-4 \times 10^{-4}$ and $J_{2}>0$, the II-type if $J_{1}<-4 \times 10^{-4}$ and $J_{2}<0$, the III-type if $J_{1}>4 \times 10^{-4}$ and $J_{2}<0$, and the IV-type if $J_{1}>4 \times 10^{-4}$ and $J_{2}>0$.

\section{APPENDIX B: COMBINATION OF LAMBERTIAN CONDITION AND SPECULAR REFLECTION}

In this appendix, we consider a free-molecular gas around a group of bodies at rest. We assume that the shape and arrangement of the bodies, as well as their surface temperature, are arbitrary. The gas may extend to infinity (the condition at infinity will be specified later). This situation is the same as in Refs. 15 and 16 and Sec. 2.5 in Ref. 4. To be consistent with these references, we use dimensional variables in this appendix. That is, $\widetilde{f}(\boldsymbol{X}, \boldsymbol{\xi})$ is the velocity distribution function, $\boldsymbol{X}$ is the position vector in space, $\boldsymbol{\xi}$ is the molecular velocity, $\xi=|\boldsymbol{\xi}|$, and $\boldsymbol{l}=\boldsymbol{\xi} / \xi ; T_{w}(\mathbf{X})$ and $n(\boldsymbol{X})$ are, respectively, the temperature and unit normal vector to the body surface $\mathrm{S}$, pointed toward the gas, at point $\boldsymbol{X}$ on the surface $(\boldsymbol{X} \in \mathrm{S})$.

In this situation, the basic equation is

$$
\boldsymbol{\xi} \cdot \frac{\partial \tilde{f}}{\partial \boldsymbol{X}}=\xi \boldsymbol{l} \cdot \frac{\partial \tilde{f}}{\partial \boldsymbol{X}}=0
$$

We assume that the boundary condition is the convex combination of the Lambertian condition [cf. Eq. (17)] and specular reflection, which can be expressed in the following form: 


$$
|\boldsymbol{\xi} \cdot \boldsymbol{n}| \widetilde{f}(\boldsymbol{X}, \boldsymbol{\xi})=\int_{\boldsymbol{\xi}_{*} \cdot \boldsymbol{n}<0}\left|\boldsymbol{\xi}_{*} \cdot \boldsymbol{n}\right| R\left(\boldsymbol{\xi}_{*} \rightarrow \boldsymbol{\xi} ; \boldsymbol{X}\right) \widetilde{f}\left(\boldsymbol{X}, \boldsymbol{\xi}_{*}\right) \mathrm{d} \boldsymbol{\xi}_{*},
$$$$
\text { for } \boldsymbol{\xi} \cdot \boldsymbol{n}>0, \quad \boldsymbol{X} \in \mathrm{S},
$$

with

$$
\begin{aligned}
R\left(\boldsymbol{\xi}_{*} \rightarrow \boldsymbol{\xi} ; \boldsymbol{X}\right)= & \alpha(\boldsymbol{X})|\boldsymbol{\xi} \cdot \boldsymbol{n}| K\left(\xi_{*}, \xi ; \boldsymbol{X}\right) \\
& +[1-\alpha(\boldsymbol{X})] \delta\left(\boldsymbol{\xi}_{*}-\boldsymbol{\xi}+2(\boldsymbol{\xi} \cdot \boldsymbol{n}) \boldsymbol{n}\right) .
\end{aligned}
$$

Here, $\alpha(\boldsymbol{X})$ is the accommodation coefficient that may depend on the position on the surface $(0<\alpha \leq 1)$ and $\delta$ is the (three-dimensional) Dirac delta function. The function $K$ is assumed to satisfy (i) non-negativity, (ii) normality, and (iii) reciprocity, i.e.,

$$
\begin{aligned}
& K\left(\xi_{*}, \xi ; \boldsymbol{X}\right) \geq 0, \\
& \pi \int_{0}^{\infty} \xi^{3} K\left(\xi_{*}, \xi ; \boldsymbol{X}\right) \mathrm{d} \xi=1, \\
& \exp \left(-\frac{m \xi_{*}^{2}}{2 k T_{w}}\right) K\left(\xi_{*}, \xi ; \boldsymbol{X}\right)=\exp \left(-\frac{m \xi^{2}}{2 k T_{w}}\right) K\left(\xi, \xi_{*} ; \boldsymbol{X}\right) .
\end{aligned}
$$

In Refs. 4 and 14-16, the Maxwell-type condition is employed, i.e.,

$$
K\left(\xi_{*}, \xi ; \boldsymbol{X}\right)=\frac{1}{2 \pi\left(k T_{w} / m\right)^{2}} \exp \left(-\frac{m \xi^{2}}{2 k T_{w}}\right),
$$

in Eqs. (B2) and (B3). In this case, we can obtain the analytical solution, which shows that the flow vanishes. We will show that the vanishing of the flow is also true for a more general boundary condition, Eqs. (B2) and (B3), though we cannot obtain an explicit solution in this case.

Now let us consider a molecule at point $\boldsymbol{X}$ in the gas with velocity $\boldsymbol{\xi}=\boldsymbol{\xi} \boldsymbol{l}$. Assuming that the molecule has reached $\boldsymbol{X}$ undergoing specular reflection on the surfaces of the bodies, we trace back its trajectory. Let $\boldsymbol{X}^{(1)}$ be the first point on the boundary $\mathrm{S}$ that we encounter, $\boldsymbol{X}^{(2)}$ be the second point, and so on (see, e.g., Fig. 2.5 in Ref. 4). If $\left|\boldsymbol{X}^{(N)}\right|=\infty$ for some $N$, the sequence $\left\{\boldsymbol{X}^{(m)}\right\}$ terminates at $m=N$. Then, we let $\boldsymbol{n}^{(m)}$ $=\boldsymbol{n}\left(\boldsymbol{X}^{(m)}\right)$ and $\alpha^{(m)}=\alpha\left(\boldsymbol{X}^{(m)}\right)(m=1,2, \ldots)$. Further, we denote by $\boldsymbol{l}^{(m)}$ the unit vector in the direction of incidence at $\boldsymbol{X}^{(m)}$, i.e.,

$$
\boldsymbol{l}^{(m)}=\boldsymbol{l}^{(m-1)}-2\left(\boldsymbol{l}^{(m-1)} \cdot \boldsymbol{n}^{(m)}\right) \boldsymbol{n}^{(m)}, \quad \boldsymbol{l}^{(0)}=\boldsymbol{l} .
$$

Note that $\boldsymbol{l}^{(m)} \cdot \boldsymbol{n}^{(m)}<0$ and $\boldsymbol{l}^{(m)} \cdot \boldsymbol{n}^{(m+1)}>0$. Then, because of Eqs. (B1)-(B3), we can express $\tilde{f}(\boldsymbol{X}, \boldsymbol{\xi})=\tilde{f}(\boldsymbol{X}, \boldsymbol{\xi} \boldsymbol{l})$ as follows:

$$
\begin{aligned}
\tilde{f}(\boldsymbol{X}, \xi \boldsymbol{l})= & \tilde{f}\left(\boldsymbol{X}^{(1)}, \xi \boldsymbol{l}^{(0)}\right) \\
= & \alpha^{(1)} F\left(\boldsymbol{X}^{(1)}, \xi\right)+\left(1-\alpha^{(1)}\right) \tilde{f}\left(\boldsymbol{X}^{(1)}, \xi \boldsymbol{l}^{(1)}\right) \\
= & \alpha^{(1)} F\left(\boldsymbol{X}^{(1)}, \xi\right)+\left(1-\alpha^{(1)}\right) \alpha^{(2)} F\left(\boldsymbol{X}^{(2)}, \xi\right)+\left(1-\alpha^{(1)}\right) \\
& \times\left(1-\alpha^{(2)}\right) \tilde{f}\left(\boldsymbol{X}^{(2)}, \xi \boldsymbol{l}^{(2)}\right) \\
= & \cdots=\sum_{m=1}^{\infty} \prod_{h=1}^{m-1}\left(1-\alpha^{(h)}\right) \alpha^{(m)} F\left(\boldsymbol{X}^{(m)}, \xi\right),
\end{aligned}
$$

where $\prod_{h=1}^{0}\left(1-\alpha^{(h)}\right)=1$ and $F(\boldsymbol{X}, \xi)$ is defined for $\boldsymbol{X} \in \mathrm{S}$ as

$$
\begin{aligned}
F(\boldsymbol{X}, \xi)= & \int_{\boldsymbol{l}_{*} \cdot \boldsymbol{n}(\boldsymbol{X})<0} \int_{0}^{\infty}\left|\boldsymbol{l}_{*} \cdot \boldsymbol{n}(\boldsymbol{X})\right| \xi_{*}^{3} K\left(\xi_{*}, \xi ; \boldsymbol{X}\right) \\
& \times \widetilde{f}\left(\boldsymbol{X}, \xi_{*} \boldsymbol{l}_{*}\right) \mathrm{d} \xi_{*} \mathrm{~d} \Omega\left(\boldsymbol{l}_{*}\right) .
\end{aligned}
$$

In addition, if $\left|\boldsymbol{X}^{(N)}\right|=\infty$ for some $N$, then the series terminates at the $N$ th term, which is replaced by

$$
\prod_{h=1}^{N-1}\left(1-\alpha^{(h)}\right) \tilde{f}\left(\infty, \xi l^{(N-1)}\right) .
$$

The truncation of the series can be made automatically if we introduce the convention $\alpha^{(N)}=1$.

Let us introduce the following quantities:

$$
\begin{aligned}
& \widetilde{g}(\boldsymbol{X}, \boldsymbol{l})=\int_{0}^{\infty} \xi^{3} \widetilde{f}(\boldsymbol{X}, \xi \boldsymbol{\xi}) \mathrm{d} \xi, \\
& \widetilde{\sigma}(\boldsymbol{X})=\int_{0}^{\infty} \xi^{3} F(\boldsymbol{X}, \xi) \mathrm{d} \xi, \quad(\boldsymbol{X} \in \mathrm{S}) .
\end{aligned}
$$

By integrating Eq. (B7) multiplied by $\xi^{3}$ with respect to $\xi$ from 0 to $\infty$, we obtain

$$
\widetilde{g}(\boldsymbol{X}, \boldsymbol{l})=\sum_{m=1}^{\infty} \prod_{h=1}^{m-1}\left(1-\alpha^{(h)}\right) \alpha^{(m)} \widetilde{\sigma}\left(\boldsymbol{X}^{(m)}\right)
$$

where, if $\left|\boldsymbol{X}^{(N)}\right|=\infty$, the series terminates at the $N$ th term, which is to be replaced by

$$
\prod_{h=1}^{N-1}\left(1-\alpha^{(h)}\right) \widetilde{g}\left(\infty, l^{(N-1)}\right) .
$$

On the other hand, if we integrate Eq. (B8) multiplied by $\xi^{3}$ with respect to $\xi$ from 0 to $\infty$ and make use of Eq. (B4b), then we have

$$
\widetilde{\sigma}(\boldsymbol{X})=\frac{1}{\pi} \int_{\boldsymbol{l}_{*} \cdot \boldsymbol{n}(\boldsymbol{X})<0}\left|\boldsymbol{l}_{*} \cdot \boldsymbol{n}(\boldsymbol{X})\right| \widetilde{g}\left(\boldsymbol{X}, \boldsymbol{l}_{*}\right) \mathrm{d} \Omega\left(\boldsymbol{l}_{*}\right),
$$

$(X \in \mathrm{S})$.

The substitution of Eq. (B11) into Eq. (B13) gives the integral equation for $\widetilde{\sigma}(\boldsymbol{X})$.

Suppose that the gas is confined in a closed domain. Then, $\widetilde{\sigma}(\boldsymbol{X})=C$, where $C$ is an arbitrary constant, is the solution of the integral equation for $\widetilde{\sigma}(\boldsymbol{X})$. In fact, if we let $\widetilde{\sigma}(\boldsymbol{X})=C$ in Eq. (B11), we have $\tilde{g}(\boldsymbol{X}, \boldsymbol{l})=C$ because $\sum_{m=1}^{\infty} \Pi_{h=1}^{m-1}\left(1-\alpha^{(h)}\right) \alpha^{(m)}=1$ holds (see Refs. 4 and 15). Then, if we let $\widetilde{g}(\boldsymbol{X}, \boldsymbol{l})=C$ in Eq. (B13), we have $\widetilde{\sigma}(\boldsymbol{X})=C$. This means that $\widetilde{\sigma}(\boldsymbol{X})=C$ is the solution. The constant $C$ is determined by the condition on the mass of the gas contained in the system.

We next consider the case where the domain of the gas extends to infinity. If we assume that $\widetilde{g}(\infty, \boldsymbol{l})=C$ with a constant $C$, then, as in the case of a closed domain, we can show that $\widetilde{\sigma}(\boldsymbol{X})=C$ is the solution of the integral equation for $\widetilde{\sigma}(\boldsymbol{X})$. Thus, the velocity distribution function $\tilde{f}(\boldsymbol{X}, \xi \boldsymbol{l})$ at infinity 
must be such that $\widetilde{g}(\infty, l)=C$. This contains the velocity distribution such as $\tilde{f}=C \beta^{2} \exp \left(-\beta \xi^{2}\right)$ (cf. Refs. 4 and 15).

Let us denote by $\rho$ the density and by $\boldsymbol{v}$ the flow velocity of the gas. Since $\widetilde{g}(\boldsymbol{X}, \boldsymbol{l})=C$ is the solution, we have

$$
\rho \boldsymbol{v}=\int \tilde{\xi} \tilde{f} \mathrm{~d} \boldsymbol{\xi}=\int \boldsymbol{l} \widetilde{g}(\boldsymbol{X}, \boldsymbol{l}) \mathrm{d} \Omega(\boldsymbol{l})=0,
$$

where the domain of integration of the first integral is the whole space of $\boldsymbol{\xi}$ and that of the second integral is all directions of $\boldsymbol{l}$. Therefore, no flow is induced in the gas, as in the case of the Maxwell-type condition. It should be noted that for the latter condition, the solution $\widetilde{f}(\boldsymbol{X}, \boldsymbol{\xi})$ can be obtained explicitly in the form of an infinite series. $2,4,14,15$

${ }^{1}$ C. Cercignani, Rarefied Gas Dynamics: From Basic Concepts to Actual Calculations (Cambridge University Press, Cambridge, 2000).

${ }^{2}$ Y. Sone, "Flows induced by temperature fields in a rarefied gas and their ghost effect on the behavior of a gas in the continuum limit," in Annual Review of Fluid Mechanics (Annual Reviews, Palo Alto, 2000), pp. 779811.

${ }^{3}$ G. E. Karniadakis and A. Beskok, Micro Flows: Fundamentals and Simulation (Springer-Verlag, New York, 2002).

${ }^{4}$ Y. Sone, Molecular Gas Dynamics: Theory, Techniques, and Applications (Birkhäuser, Boston, 2007).

5 J. C. Maxwell, "On stresses in rarefied gases arising from inequalities of temperature," Philos. Trans. R. Soc. London 170, 231 (1879).

${ }^{6}$ E. H. Kennard, Kinetic Theory of Gases (McGraw-Hill, New York, 1938).

${ }^{7}$ Y. Sone, "Thermal creep in rarefied gas," J. Phys. Soc. Jpn. 21, 1836 (1966).

${ }^{8}$ T. Ohwada, Y. Sone, and K. Aoki, "Numerical analysis of the shear and thermal creep flows of a rarefied gas over a plane wall on the basis of the linearized Boltzmann equation for hard-sphere molecules," Phys. Fluids A 1, 1588 (1989).

${ }^{9}$ Y. Sone, "Flow induced by thermal stress in rarefied gas," Phys. Fluids 15, 1418 (1972).

${ }^{10} \mathrm{~T}$. Ohwada and Y. Sone, "Analysis of thermal stress slip flow and negative thermophoresis using the Boltzmann equation for hard-sphere molecules," Eur. J. Mech. B/Fluids 11, 389 (1992).

${ }^{11}$ M. N. Kogan, V. S. Galkin, and O. G. Fridlender, "Stresses produced in gases by temperature and concentration inhomogeneities. New type of free convection," Sov. Phys. Usp. 19, 420 (1976).

${ }^{12}$ K. Aoki, Y. Sone, and N. Masukawa, "A rarefied gas flow induced by a temperature field," in Rarefied Gas Dynamics: 19th International Symposium, edited by J. Harvey and G. Lord (Oxford University Press, Oxford, 1995), pp. 35-41.

${ }^{13}$ Y. Sone and M. Yoshimoto, "Demonstration of a rarefied gas flow induced near the edge of a uniformly heated plate," Phys. Fluids 9, 3530 (1997).

${ }^{14}$ Y. Sone, "Highly rarefied gas around a group of bodies with various temperature distributions. I. Small temperature variation," J. Méc. Théor. Appl. 3, 315 (1984).

${ }^{15}$ Y. Sone, "Highly rarefied gas around a group of bodies with various temperature distributions. II. Arbitrary temperature variation," J. Mec. Theor. Appl. 4, 1 (1985).

${ }^{16}$ Y. Sone, "Boundary temperature effect in a highly rarefied gas," Phys. Fluids 28, 419 (1985).

${ }^{17}$ K. Aoki, C. Bardos, F. Golse, M. N. Kogan, and Y. Sone, "Steady flows of a rarefied gas around arbitrary obstacle distributions," Eur. J. Mech. B/Fluids 12, 565 (1993).

${ }^{18}$ C. Cercignani, A. Frezzotti, and M. N. Kogan, "On the absence of motion in certain nonequilibrium states of gases and vapors in free-molecular regime: General considerations and pipe flow," Phys. Fluids A 5, 2551 (1993).

${ }^{19} \mathrm{~S}$. Nocilla, "On the interaction between stream and body in free-molecule flow," in Rarefied Gas Dynamics: Second International Symposium, edited by L. Talbot (Academic, New York, 1961), pp. 169-208.

${ }^{20}$ M. Epstein, "A model of the wall boundary condition in kinetic theory," AIAA J. 5, 1797 (1967).

${ }^{21}$ I. Kuščer, J. Možina, and F. Krizanic, "The Knudsen model of thermal accommodation," in Rarefied Gas Dynamics: Seventh International Symposium, edited by D. Dini (Editrice Tecnico Scientifica, Pisa, 1971), Vol. 1 , pp. 97-108.

${ }^{22}$ C. Cercignani and M. Lampis, "Kinetic models for gas-surface interactions," Transp. Theory Stat. Phys. 1, 101 (1971).

${ }^{23} \mathrm{C}$. Cercignani, "Models for gas surface interactions: Comparison between theory and experiment," in Rarefied Gas Dynamics: Seventh International Symposium, edited by D. Dini (Editrice Tecnico Scientifica, Pisa, 1971), Vol. 1, pp. 75-95.

${ }^{24}$ C. Cercignani, The Boltzmann Equation and Its Applications (SpringerVerlag, New York, 1988).

${ }^{25}$ C. Cercignani, "Free molecular flow past a flat plate in the presence of a nontrivial gas-surface interaction," Z. Angew. Math. Phys. 23, 713 (1972).

${ }^{26}$ F. Sharipov, "Application of the Cercignani-Lampis scattering kernel to channel gas flows," in Rarefied Gas Dynamics: 22nd International Symposium, edited by T. J. Bartel and M. A. Gallis (AIP, Melville, 2001) [AIP Conf. Proc. 585, 347 (2001)].

${ }^{27}$ C. E. Siewert, "Generalized boundary conditions for the S-model kinetic equations basic to flow in a plane channel," J. Quant. Spectrosc. Radiat. Transf. 72, 75 (2002).

${ }^{28}$ C. Cercignani, M. Lampis, and S. Lorenzani, "Plane Poiseuille flow with symmetric and nonsymmetric gas-wall interactions," Transp. Theory Stat. Phys. 33, 545 (2004).

${ }^{29}$ R. F. Knackfuss and L. B. Barichello, "Surface effects in rarefied gas dynamics: An analysis based on the Cercignani-Lampis boundary condition," Eur. J. Mech. B/Fluids 25, 113 (2006).

${ }^{30}$ G. A. Bird, "Approach to translational equilibrium in a rigid sphere gas," Phys. Fluids 6, 1518 (1963).

${ }^{31}$ G. A. Bird, Molecular Gas Dynamics and the Direct Simulation of Gas Flows (Oxford University Press, Oxford, 1994).

${ }^{32}$ R. G. Lord, "Some extensions to the Cercignani-Lampis gas-surface scattering kernel," Phys. Fluids A 3, 706 (1991).

${ }^{33}$ M. Abramowitz and I. A. Stegun, Handbook of Mathematical Functions (Dover, New York, 1968).

${ }^{34}$ To be more specific, the back scattering is a scattering in which $\left[\boldsymbol{\zeta}_{*}\right.$ $\left.-\left(\boldsymbol{\zeta}_{*} \cdot \boldsymbol{n}\right) \boldsymbol{n}\right] \cdot[\boldsymbol{\zeta}-(\boldsymbol{\zeta} \cdot \boldsymbol{n}) \boldsymbol{n}]<0$ holds, where $\boldsymbol{\zeta}_{*}$ and $\boldsymbol{\zeta}$ are molecular velocities before and after the reflection and $\boldsymbol{n}$ is the unit normal vector to the boundary.

${ }^{35}$ R. G. Lord, "Application of the Cercignani-Lampis scattering kernel to direct simulation Monte Carlo calculations," in Rarefied Gas Dynamics: 17th International Symposium, edited by A. E. Beylich (VCH, Weinheim, 1991), pp. 1427-1433.

${ }^{36}$ T. C. Lilly, J. A. Duncan, S. L. Nothnagel, S. F. Gimelshein, N. E. Gimelshein, A. D. Ketsdever, and I. J. Wysong, "Numerical and experimental investigation of microchannel flows with rough surfaces," Phys. Fluids 19, 106101 (2007)

${ }^{37}$ A. D. Ketsdever, M. T. Clabough, S. F. Gimelshein, and A. Alexeenko, "Experimental and numerical determination of micropropulsion device efficiencies at low Reynolds numbers," AIAA J. 43, 633 (2005). 